\title{
Accessible Review of Internet of Vehicle Models for Intelligent Transportation and Research Gaps for potential Future Directions
}

\author{
Sakshi Garg${ }^{1}$, Deepti Mehrotra², Hari Mohan Pandey ${ }^{3}$, Sujata Pandey ${ }^{4}$
}

\begin{abstract}
This paper presents a state-of-the-art review of various models of Internet of Vehicles (IoV) that were proposed to revolutionize the transportation and automation industry. In the last few years, IoV technology has emerged as a global revolution, demonstrating considerable impact upon the transportation and automation industry, hence, the interest of the researchers bends towards the utility of the IoV technology which motivated us to present a comprehensive review. This paper, thus, collates recent findings along with some anticipated applications of IoV technologies for transportation. It brings to light the evolution of the IoV studies referred as models for transportation since its birth and aims to present a deep insightful review of various IoV technologies with their strengths and weaknesses. The reviewed literature is organized systematically to give a categorical overview of recent developments. This article also discusses the outstanding potentialities and challenges that exist in transportation from IoV perspective with the belief that these will have the capacity to be addressed in the near future. As there is no review literature on IoV technology available for transportation. Hence, we strongly believe that this study will be useful and enable the researchers to utilize IoV technologies to solve complex outstanding problems in transportation and automation.
\end{abstract}

Keywords Internet of Vehicles, V2X, Network, Energy efficiency, Big data, QoS.

\section{Introduction}

Internet of Things (IoT) [1] is a revolution for the current era, from applications that connect computing devices, digital and mechanical devices, objects, to people who possess unique identifiers. IoT has shown its ability to transmit data over a network without needing human interaction at any level [2]. In smart transportation, IoT has altered (e.g. trucking, automation [3], transportation [4], etc.) industry entirely with the aid of mobile and internet connectivity advancements. Some salient features of IoT in transportation are fleet management and public transit management [5], smart inventory management [6], optimal asset utilization and geo-fencing [7], etc. Over the past few years, a naïve interrelated concept came in the frame, which is referred as "Internet of Vehicles $(\mathrm{IoV})^{1}{ }^{1}$ ". IoV is transforming the transportation system into a globally diverse network [8].

\footnotetext{
Sakshi Garg. sakshijyotigarg@gmail.com [0000-0001-6467-9065]

Deepti Mehrotra. mehdeepti@gmail.com [0000-0001-5752-9800]

Hari Mohan Pandey. profharimohanpandey@gmail.com [00000002-9128-068X]

Sujata Pandey. spandey@amity.edu [00000003-2669-8323]
}

\footnotetext{
${ }^{1} \mathrm{IoV}$ is abbreviated as Internet of Vehicles which has also been referred to by various names like ICV (Internet Connected Vehicles) or CV (Connected Vehicles) or AUV (Autonomous Vehicles) by other academicians.
}

1 Department of Computer Science and Engineering, Amity University, Uttar Pradesh, India.

2 Department of Information Technology, Amity University, Uttar Pradesh, India

3 Department of Computer Science, Edge Hill University, Ormskirk, United Kingdom.

4 Department of Electronics and Communication Engineering, Amity University, Uttar Pradesh, India.

Broadly, the IoV is an integration of three networks as shown in figure 1: an inter-vehicle network, an intra-vehicle network, and the vehicular mobile Internet. [9-10].

IoV encourages the concordant unification of people, vehicles, their streets, and the surroundings. This system accumulates data about the position, movement of vehicles, etc. and provides these data to other connected networks and vehicles on the road for communication and assistance. This also opens new channels for communication services [11] for big smart cities, varying from traffic monitoring [12], safety applications [13], to gathering of data [14]. IoV technology also comprises the installation of Artificial Intelligence (AI) in future systems to affix administration conveyance and activities, influence Quality of Service (QoS) and assurance administration, accessibility, additionally permitting better deftness, versatility, quicker customization and security. In any case, the IoV is considered as an essential image of the Smart City idea and it has a wide application prospect in building future Intelligent Transportation System where various factors like network efficiency, cost and energy efficiency [15],

\footnotetext{
${ }^{2} \mathrm{~V} 2 \mathrm{X}$ represents $\mathrm{V} 2 \mathrm{~V}, \mathrm{~V} 2 \mathrm{I}, \mathrm{V} 2 \mathrm{H}, \mathrm{V} 2 \mathrm{R}$ and $\mathrm{V} 2 \mathrm{C}$ which is also referred to as $\mathrm{V} 2 \alpha$ by other academicians.
} 
standardization, big data handling, privacy and security [16] still should be handled to permit a superior use of IoV [17]. However, this requires extensive data analysis, determination of factors dependent and affecting IoV with

Fig. 1 Internet of Vehicle Classification constant need of experiments and simulations to test the data accumulated and analyzed to provide sustainable and reliable information to other dependent sources using it. The simulations and experiments conducted by far further needs arduous improvement and advancement [18].

\section{INTERNET OF VEHICLES}

The contributions of this study are as follows:

1. The paper presents the rigorous analysis of the factors affecting IoV along with related study and proposed methods with their pros and cons for future directions.

2. The future works of the simulation and experimental studies have been mentioned for the researchers and readers to carry their further studies.

3. It clearly mentions the research gaps existing till date from the time of evolution to present and describes the strengths and weaknesses of the gaps and its approaches which will provide clear directions for further studies in IoV for future.

The paper is further divided into 5 sections: Section II gives an overview of the State-of-art and review methodology considered in further sections. Sub-sections of Section II describe comprehensively the Factors affecting the Internet of Vehicles and Theoretical Framework proposed in the literature till date. Section III talks about the Research Gaps and their analysis based on the above study and the recent research trends in this field. Section IV presents tools used in the field of $\mathrm{IoV}$ for simulation in tabular form and the last section $\mathrm{V}$ gives the conclusion and future scope for the entire study.

\section{State-of-Art of loV and Review Methodology}

The long standing of $\mathrm{IoV}$ is a system of inter-related vehicles that can drive autonomously by using data from the roadside and other vehicles on the road. IoV utilizes the beauty of Artificial Intelligence (AI) with some sort of automation. Since, we see IoV as a burning topic, we present a state-of-art of the existing reviewed and

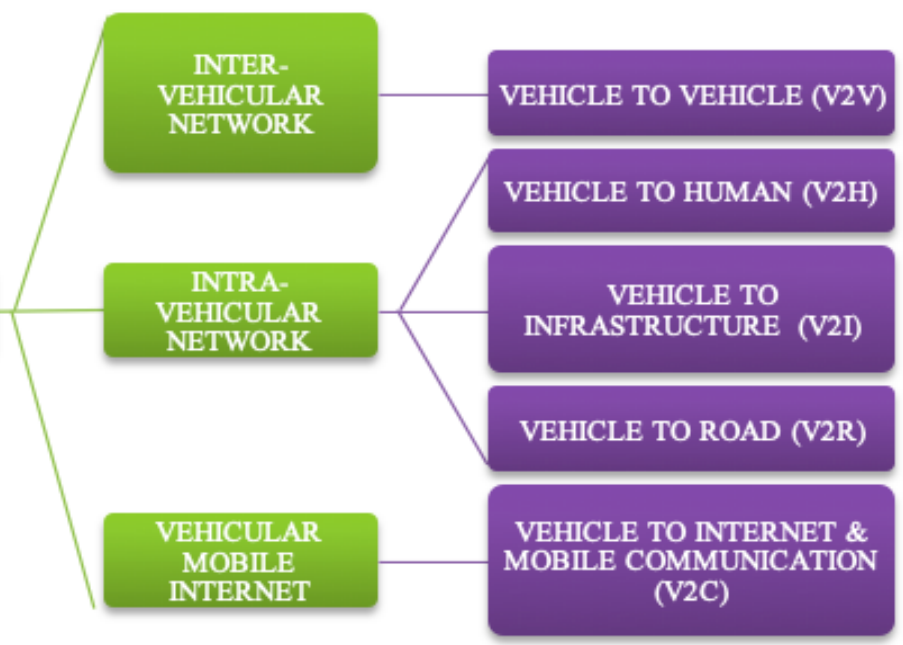

experimented literature studies. Figure 2 represents the graph showing the timeline of IoV. It shows the number of studies considered throughout this review. The graph presents the state of art for IoV from 1999 to till date. However, studies from 1999 to 2009 are very few, hence they have been added together under 'Before 2010' marking. It is well evident from the graph that with the growing years, IoV has been of keen interest for more and more researchers. Also, this interpretation will encourage academicians and researchers to explore and advance this field to newer possibilities.

For this purpose, we have explored all esteemed libraries and datasets like ACM, Springer, Elsevier, IEEE, etc. We have taken into consideration all the reputed journals like IEEE transactions, IEEE Access, Emerging Technologies for Connected Internet of Vehicles and Intelligent Transportation System Networks, etc. and conferences like Vehicular Technology Conference, International Conference of Vehicular Technology, etc. to prepare our state-of-art in order to provide one stop discussion for most of the researches till date in the field of IoV. The papers that we have studied during this study are of different domains. Some had focused on discussing network issues in IoV, others had talked about the privacy and secrecy necessity for IoV. But we couldn't find a study that could bring out and justify the evaluation of IoV as a research field till date. Hence, this motivated us to write this article. We have then, shortlisted 193 papers which are most relevant to this study. Further, we have segregated these papers into review and experimental. Review papers along with their strengths and weaknesses are discussed in "Factors affecting the Internet of Vehicles" sub-section. Based on the factors analyzed in this section, we have discussed the experimental and numerical works in "Theoretical Framework" sub-section. This as whole has given us the state-of-art for the IoV study, through which we have further highlighted the research gaps found during these studies. Then, we have summarized the literature review in terms of simulators considered for this study. Lastly, we have presented the conclusion along with the future scope for this study. The flow of this paper is shown in figure 3 . 
Fig. 2 IoV Timeline: Growth of IoV during these years.

Fig. 3 Flow of the Paper
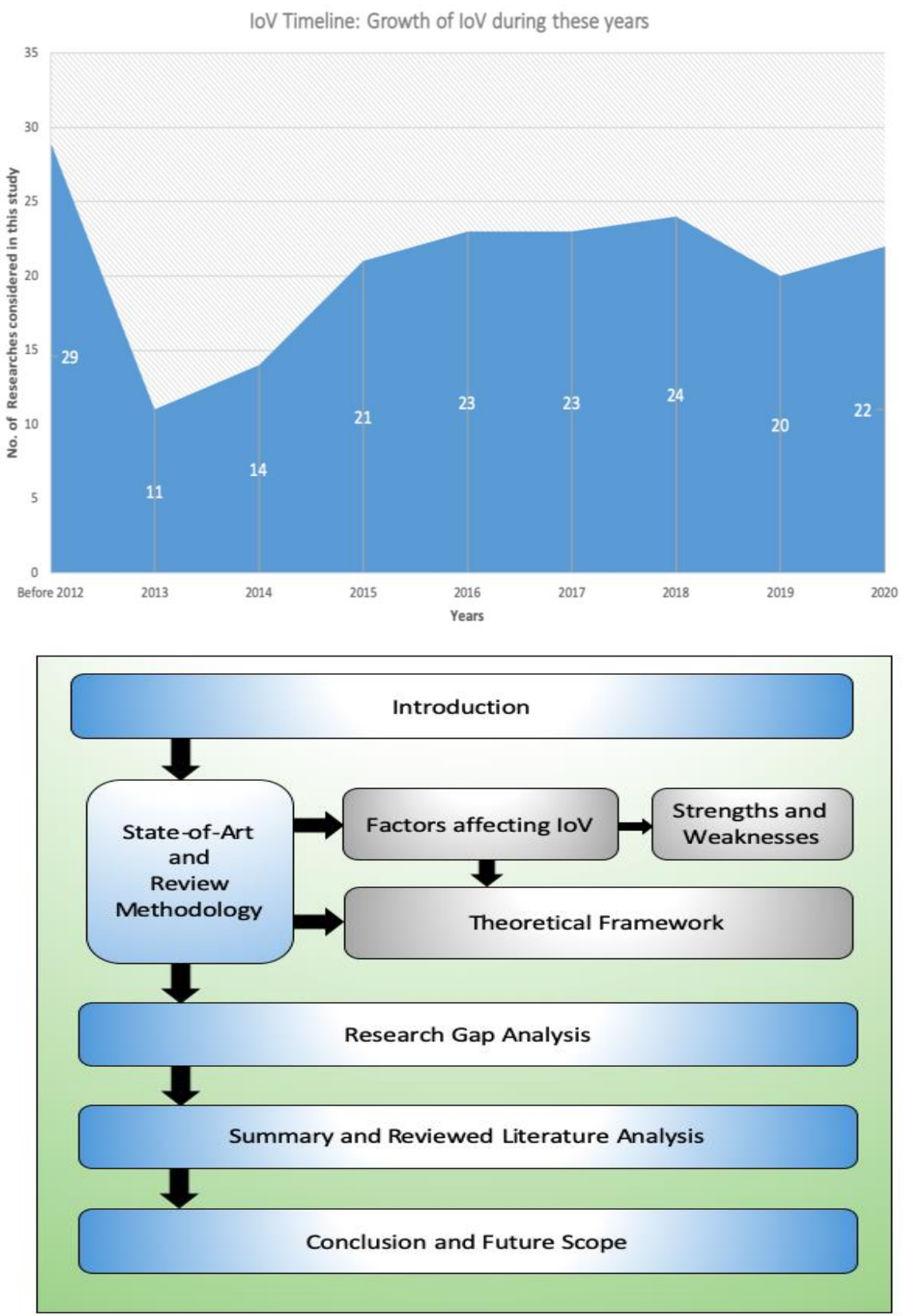

\subsection{Factors Affecting the Internet of Vehicles}

The quintessence of IoV lies in settling the communication of vehicles with the road, human and among vehicles in the present environment endowed with more transportation efficiency, diminution in the social cost and superior standards of services for cities. The experts of the Institute of Electrical and Electronics Engineers (IEEE) claim that $75 \%$ of the total road traffic will be covered by autonomous cars [19] by the year 2040 .

However, before discussing the factors one needs to clarify that IoV is not the same as VANET since IoV is often mistaken as VANET. The fact is, IoV is concerned primarily with the road map and characteristics pertaining to large cities like traffic jams, tall buildings, bad driver behaviors, larger mobile networks, and complex road networks as depicted in figure 4 , whereas, VANET involves objects that are temporary and unstable, covers a comparatively smaller network and does not provide global and sustainable applications or services to customers. Therefore, IoV is altogether a new domain in the field of Network Intelligence. Hence, we discuss the factors affecting IoV: Network, Infrastructure and Cost, Data Analysis, Standards and Service Providers, Privacy and Security.

\subsubsection{Network}

IoV basically needs correct network deployment for becoming fully functional. Various factors like network partitions, route failures, routing protocols, channel quality, data rate, network load, Software Defined Networking (SDN) [20-21], Information-Centric Networking (ICN), Content-Centric Networking (CCN) [22-23] to Named Data Networking (NDN) [24-25] are important features of Vehicular Networking. 
Authors like Yang [26], Singh and Singh [27], Jiau et al. [28], Amadeo et al. [29-30], Senouci et al. [31], Hussain et al. [37] had discussed future directions in respect with the impact of these factors on the network. Wan et al. [32] had also discussed the edge computing using 5G technology with respect to IoV. Though, deploying $5 \mathrm{G}$ enabled IoV still presents a considerable test in real scenario. Seamless switching, interference management, computational speed are some of the other open issues still to be addressed. With the growing impact of the network, a new sub-domain of IoV emerged called Social IoV (SIoV) where vehicles are treated as social entities in the machine-to-machine (M2M) vehicular social networks. Roadspeak, NaviTweet, Caravan Track are some of the emerging technologies in this area. Maglaras et al. [33], Siddiqa et al. [34] had presented the issues like network deployment in SIoV, big- data pre-processing, privacy and security as major challenges pertaining to implementation of SIoV on the network platform.

Zhang and $\mathrm{Xi}$ [35], had explained the network in IoV as "three horizontals, three verticals, and one mode". Three horizontals include standardization in the intelligent workstation, completing and integrating the technology research and the network integrated communication in big data and cloud platforms. Three verticals contain the integration of the information to ensure energy saving and vehicle safety, information services and intelligent manufacturing. One mode aims at developing the innovative business model and enhancing industrial cooperation. Thus, strengths, weaknesses and considered factors of some of the key research papers in the network domain are discussed in Table 1 for better understanding.

Fig. 4 Roadmap depiction of Internet of Vehicles

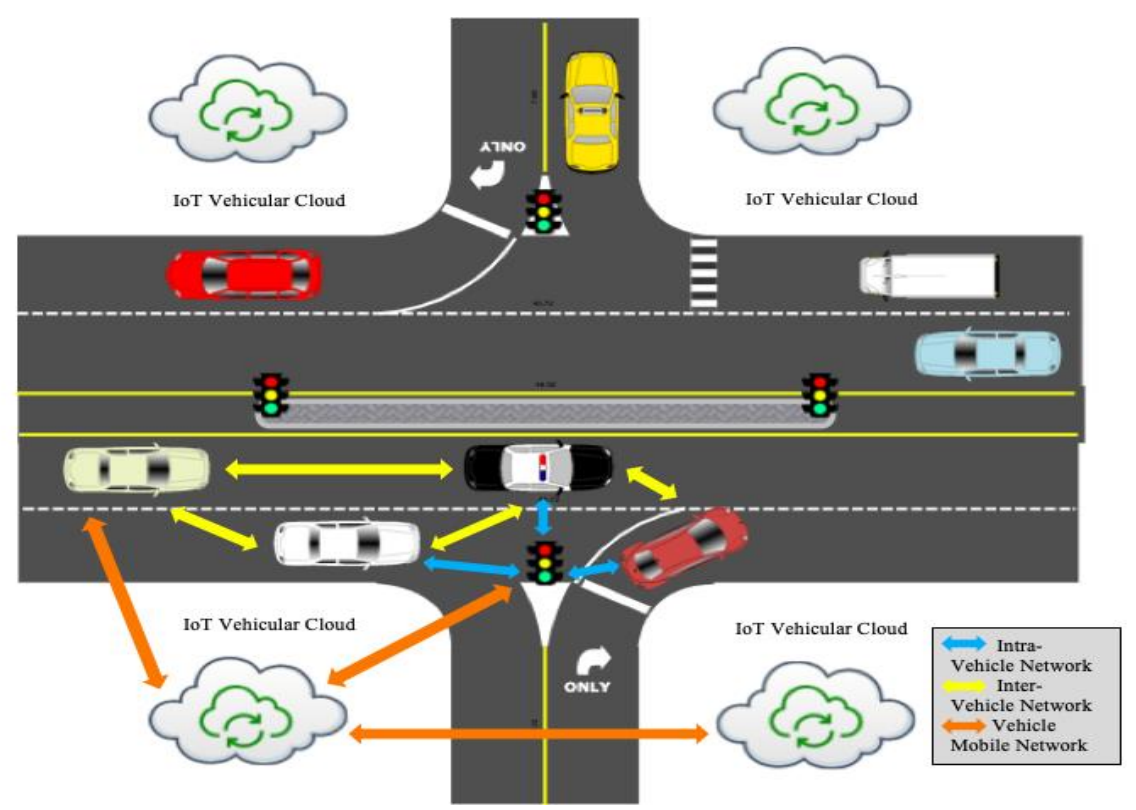

Table 1 Major factors affecting network discussed by some of the authors

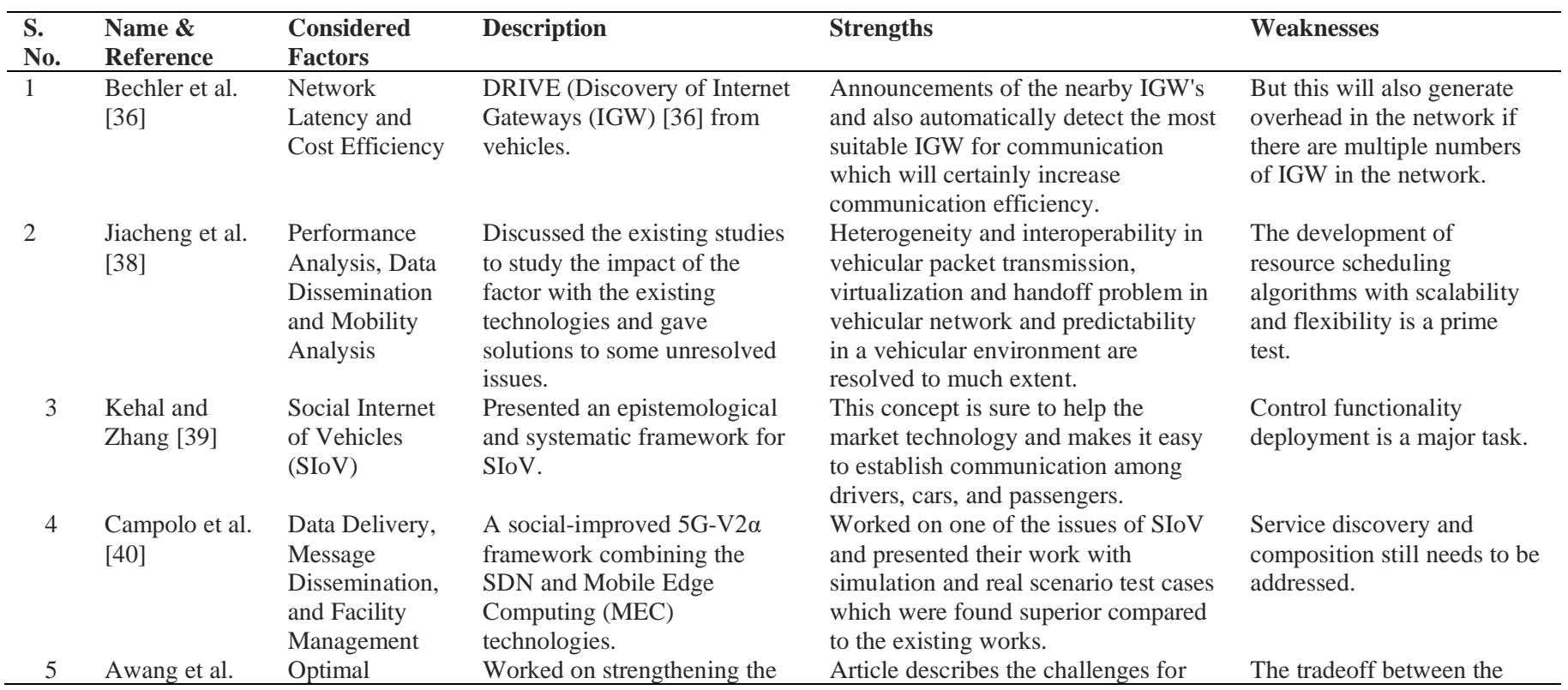




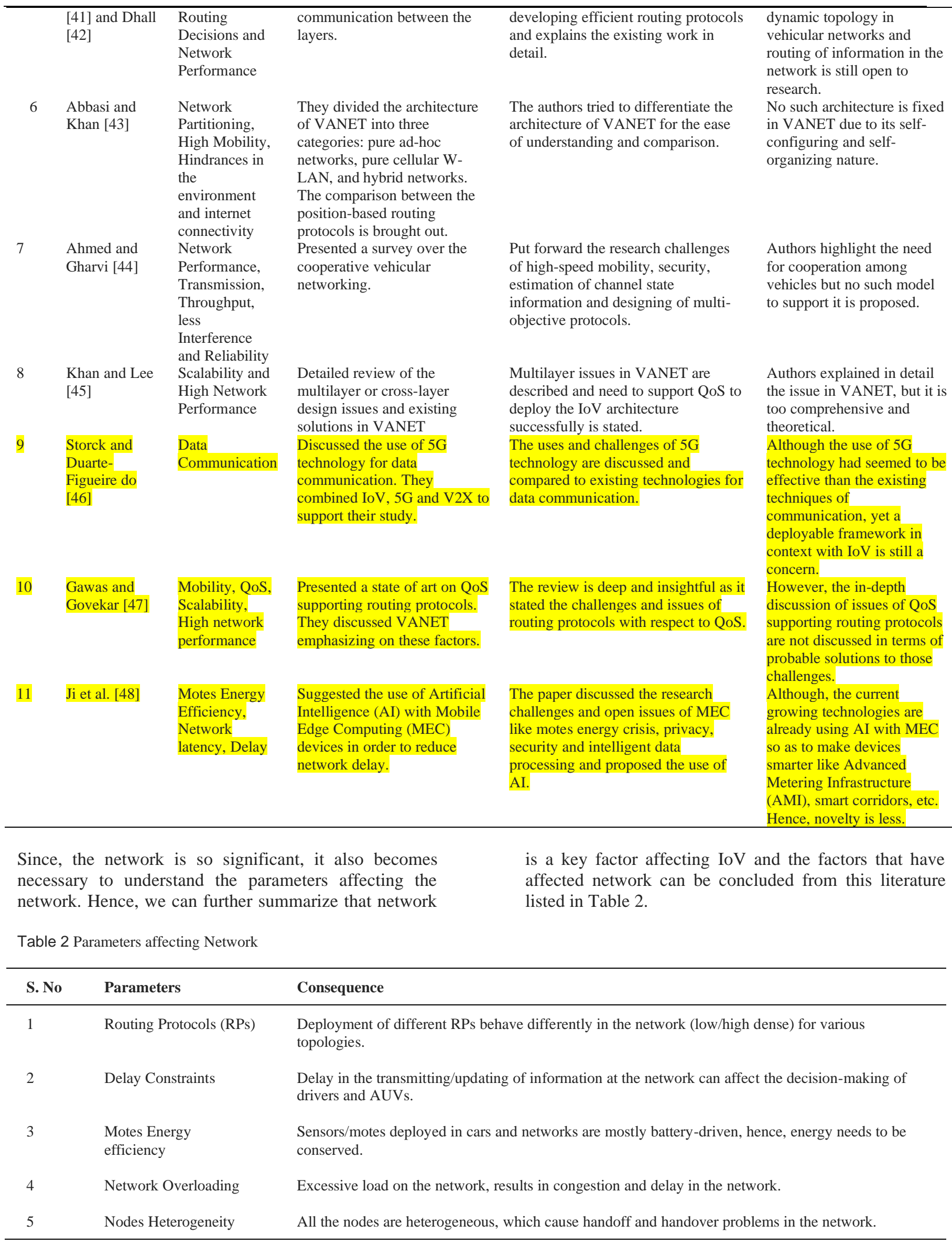




\subsubsection{Infrastructure and Cost}

IoV setup with minimum cost and reliable infrastructure plays a key role. The need for improved spectrum efficiency with enhanced Cognitive Radio (CR) technology for IoV related studies [49-52] came into light. Chembe et al. [53] had discussed the issues like infrastructure [54], deployment in the network and potential research directions in Cognitive Vehicular Network in detail. Kuutti et al. [55] had tried to deepen the knowledge base by reviewing the localization techniques to establish the infrastructure that can possibly be used in
IoV. Hence, one can conclude from the study that lack of fully developed infrastructure and hefty cost involvement had affected the growth of IoV reversely. Therefore, it is mandatory to develop such techniques and technology that can support infrastructure with low cost so as to make it feasible and accessible for everyone. Although some researchers had been working on this challenge but still it needs to be tackled in superior ways. Some of such works in favor had been discussed in Table 3.

Table 3 Major factors affecting infrastructure and cost discussed by some of the authors

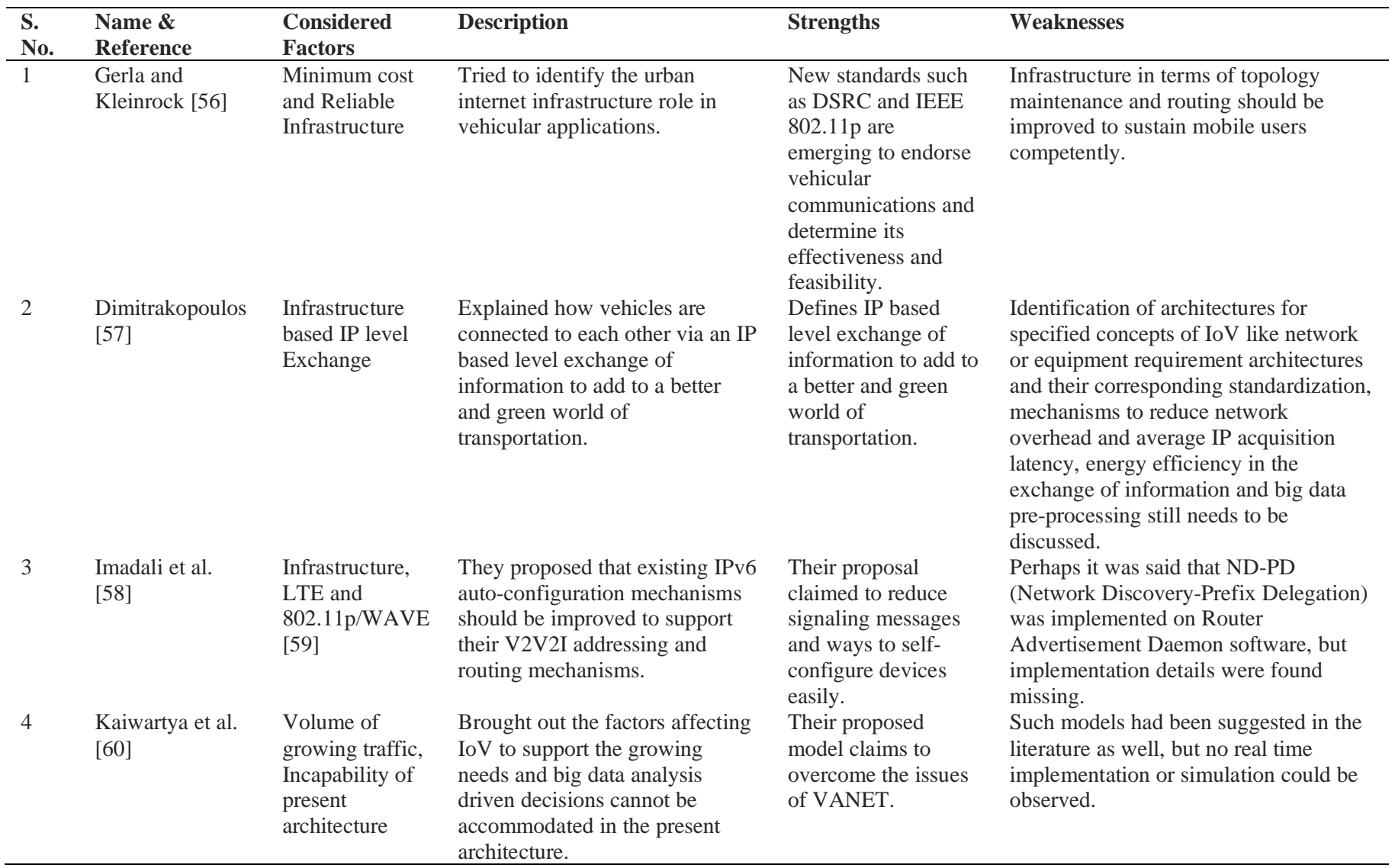

Also, we need to acknowledge the parameters affecting the infrastructure and cost so as to explore the field in every perspective. Therefore, we concluded those parameters from our study of literature and are represented in Table 4.

Table 4 Parameters affecting Infrastructure and Cost

\begin{tabular}{lll}
\hline S. No & Parameters & Consequence \\
\hline 1 & Resource Management & Proper infrastructure needs availability and accessibility to maximum resources. \\
2 & Cost Efficiency & $\begin{array}{l}\text { Hefty cost investment in defining the infrastructure for IoV has to be made economical for easier } \\
\text { accessibility. }\end{array}$ \\
3 & Traffic Assessment & $\begin{array}{l}\text { Traffic patterns are never scalable for IoV which needs to assess and monitor the traffic for better } \\
\text { infrastructure support. }\end{array}$ \\
4 & Topology maintenance & No fixed nodes topologies make it difficult to lay down a fixed infrastructure.
\end{tabular}




\subsubsection{Data Analysis}

The amount of data being generated every second is making analysis of data necessary to ensure the saving of only necessary information among the entire content. Subsequently, a white paper on the Internet of Vehicles in 2014 by China [61] explained that massive data or 'Big Data' needs rigorous pre-processing since all the other problems will be solved half way if the data being generated every day exponentially is processed properly before actually feeding it to the network for usage. For this purpose, authors like Hossain et al. [62], Chao and Yanmin [63], Yuan et al. [64], $\mathrm{Hu}$ et al. [65] had studied the QoS requirements to ensure efficient and timely data transmission in vehicular networks. Some works of authors in this regard as listed in Table 5 .

Table 5 Major factors affecting data analysis discussed by some of the authors

\begin{tabular}{|c|c|c|c|c|c|}
\hline S. No. & $\begin{array}{l}\text { Name \& } \\
\text { Reference }\end{array}$ & Considered Factors & Description & Strengths & Weaknesses \\
\hline 1 & Datta et al. [66] & $\begin{array}{l}\text { Seamless Interoperability } \\
\text { Support, Information- } \\
\text { centric Mobile Integration, } \\
\text { Mobile Edge Computing } \\
\text { Integration, Lack of data- } \\
\text { oriented Networking, Lack } \\
\text { of cloud and Data } \\
\text { Interoperability. }\end{array}$ & $\begin{array}{l}\text { Presented the engineering } \\
\text { challenges related to data } \\
\text { analysis }[67][68][69] \text {. }\end{array}$ & $\begin{array}{l}\text { The literature suggests that } \\
\text { IoT architecture is } \\
\text { incapable of } \\
\text { accommodating IoV } \\
\text { components. }\end{array}$ & $\begin{array}{l}\text { The existing technologies had to be } \\
\text { revised in order to incorporate with } \\
\text { IoV. }\end{array}$ \\
\hline 2 & $\begin{array}{l}\text { Pourghebleh and } \\
\text { Navimipour [70] }\end{array}$ & $\begin{array}{l}\text { Data Aggregation, Data } \\
\text { Transmission, Packet Loss, } \\
\text { Delivery Rate, Vehicle } \\
\text { Density. }\end{array}$ & $\begin{array}{l}\text { Gave further insight into data } \\
\text { collection mechanisms in } \\
\text { VANET's. }\end{array}$ & $\begin{array}{l}\text { Article discussed the data } \\
\text { collection schemes in } \\
\text { detail and the need for such } \\
\text { schemes. }\end{array}$ & $\begin{array}{l}\text { They presented the need to detect } \\
\text { the relevant, needed and expected } \\
\text { information to broadcast with } \\
\text { respect to the real traffic as the } \\
\text { future scope that requires extensive } \\
\text { data analysis, but no such } \\
\text { examples were referred in support. }\end{array}$ \\
\hline 3 & Gerla et al. [71] & $\begin{array}{l}\text { High Mobility, QoS } \\
\text { Requirements, Machine } \\
\text { learning for Optimization. }\end{array}$ & $\begin{array}{l}\text { Discussed the issues pertaining } \\
\text { to IoV for big-data and } \\
\text { suggested the use of } \\
\text { reinforcement learning } \\
\text { approach for optimizing the } \\
\text { network. }\end{array}$ & $\begin{array}{l}\text { Discussed the issues like } \\
\text { location-based routing, } \\
\text { load balancing and vertical } \\
\text { hand-off problem for IoV } \\
\text { in terms of Big-Data. }\end{array}$ & $\begin{array}{l}\text { The open challenges like security } \\
\text { issues, distributed learning, etc. are } \\
\text { discussed in broad. No in-depth } \\
\text { details like what other studies had } \\
\text { been stated and presented in } \\
\text { literature to overcome such issues. }\end{array}$ \\
\hline 4 & Lee et al. [72] & $\begin{array}{l}\text { Big Data, Cellular } \\
\text { Communications, Cloud } \\
\text { Computation. }\end{array}$ & $\begin{array}{l}\text { Discussed DSRC technique } \\
\text { and compared it to other } \\
\text { cellular techniques with the } \\
\text { aim to highlight the challenges } \\
\text { in Cloud computation and big } \\
\text { data pre-processing. }\end{array}$ & $\begin{array}{l}\text { DSRC versus other cellular } \\
\text { techniques are well } \\
\text { compared which gives a } \\
\text { clear picture of the issues I } \\
\text { cloud computing pertaining } \\
\text { to Big-Data. }\end{array}$ & $\begin{array}{l}\text { Big data is an open challenge } \\
\text { which is discussed only in context } \\
\text { of cloud computation. No } \\
\text { prospective solutions are stated } \\
\text { and other areas of Big-Data like } \\
\text { pre-processing, data acquisition, } \\
\text { etc. could had also been explored. }\end{array}$ \\
\hline
\end{tabular}

Apart from these factors, another interesting progression of intelligent vehicle grid to self-governing and internetconnected vehicles through Vehicular Cloud in respect to data analysis [73] is observed. Autonomous Vehicles (AUV) [74-76] is considered as potential solutions to various unprocessed Big-Data [77], network congestion and pollution issues [78]. Academicians [79-82] had made efforts to develop new interfaces that do not rely on the driver's vision as in the case with conventional screenbased interfaces to reduce the amount of data generated every second in respect to this setup. A detailed survey over AUV in expediting the application over the smart highway regarding data is presented by Okuda et al. [83], Jo et al. [84], Hussain and Zeadally [85] and Hamid et al. [86].

Further, we can say that big data accounts for deep web analysis before being stored and also that the factors affecting data analysis become important. Thus, parameters concluded by us from our study are Big-data Preprocessing, Big-data to Cloud Interaction, Big-data
Transmission, Big-data Integration, Big-data Dissemination and Big-data Storage.

\subsubsection{Standard and Service Providers}

IoV will promote the integration of automotive and information technology that shall reduce the lack of coordination and communication among vehicles and improve products, services, and experiences. For instance, Sadiku et al. [87], had discussed various technologies like wired, wireless, sensing, etc. that make use of Intelligent Transportation System (ITS), components of ITS like ramp meters, highway advisory radios, etc., its benefits and challenges. However, no vehicular models that deploy these technologies are considered. Researchers [88-91] had tried to enhance the Dedicated ShortRange Communications (DSRC) performance through PHY (Physical) and MAC (Medium Access Control) layer optimizations. Huang [92] had discussed the standards and key technologies to facilitate IoV service providers and elucidated the applications using these technologies at present. Lu et al. 
[93] had presented a detailed overview of the V2V, V2R, and V2I infrastructure connectivity. They defined the characteristics and challenges associated with each infrastructural technology like Bluetooth, ZigBee, RFID, Ultra-Wide Band, and $60 \mathrm{GHz}$ Millimeter wave for V2R, VANET, DSRC and Dynamic Spectrum Access (DSA) for V2V and Drive-Thru Network and Wi-Fi for V2I. Wu et al. [94], had explained IoV as a hybrid system with both peer-2-peer and client-server computing paradigms. They listed the characteristics and challenges of IoV explaining the major factors affecting IoV like dynamic topology, network density, geographical communications, mobility, delay constraints, scalability requirements, and privacy and security [95][96].

However, we could infer from our literature study that the chief technologies used at present are Radio Frequency Identification (RFID), sensors and DSRC. RFID is used for parking and pipeline monitoring, so this technology in IoV can offer users positioning, navigation and electronic map. Floating Car Data technology by Intelligent Support Systems (ITS) is one of the recent advances in this field. Electronic Toll Collection (ETC) uses DSRC technology for communication between vehicles and toll stations for fully automated toll collection without needing the vehicles to stop at the toll station pay. This will surely decrease the network overhead to ensure communication between Road-Side Unit (RSU) and On-Board unit (OBU) through DSRC but the installation cost will increase to a larger extent because OBD has to be installed in every vehicle to make this suggested system successful, which is practically not possible. Traffic Guidance System (TGS), Parking Management System (PMS), Public Traffic Management system (PTMS), Traffic Monitoring System (TMS) [97] are some of the applications of IoV technology. Figure 5 also shows an instance of the TMS.

\section{Fig. 5 Traffic Monitoring System}

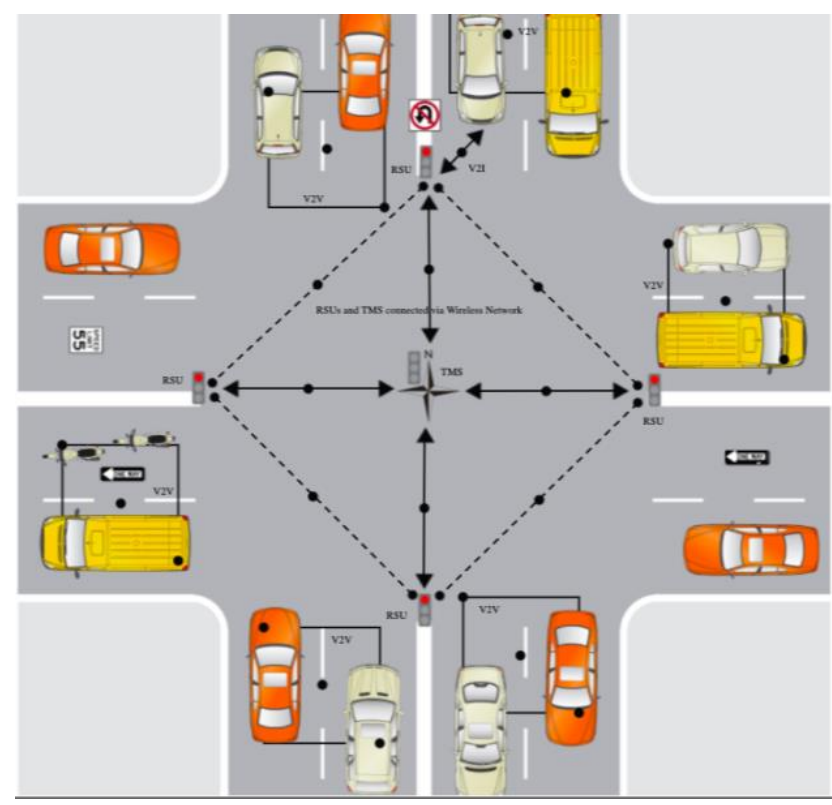

Also, we could see a major problem in specifying standards of $\mathrm{IoV}$ is due to no fixed architecture of $\mathrm{IoV}$ and its technologies. Pavan et al. [98] in 2020 had also discussed the issue of no fixed IoV architecture, its layer and implementation but no major advancements could be seen from the study. Hence, we present our generalized system architecture of IoV in figure 6 which is subjective to changes as per consideration by the researchers' propositions since no fixed structure is available. 


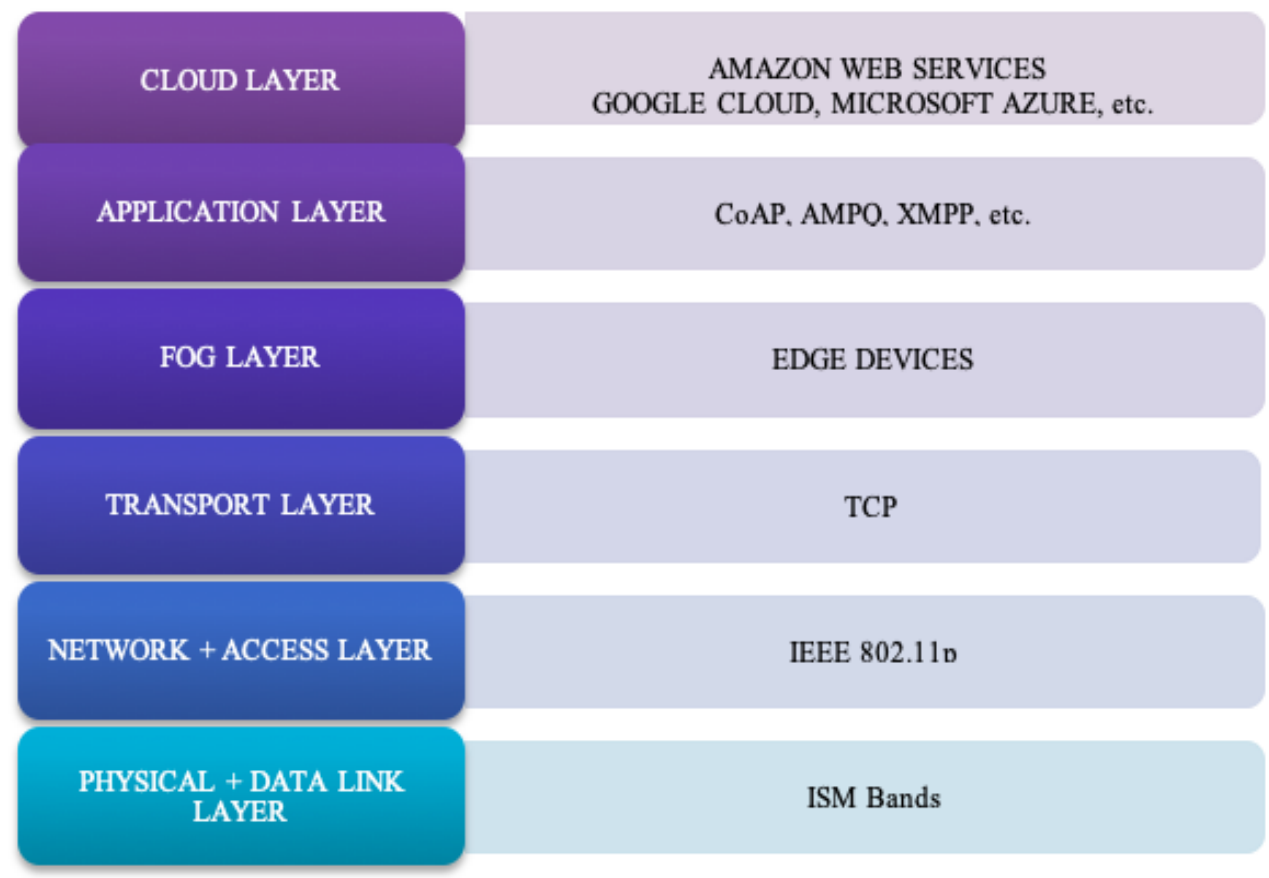

However, we had withdrawn some of the parameters we have found that affect the standards and service providers are: No IoV specific Technologies, No fixed Architecture, Scalability Requirements, QoS Requirements and Spectrum Resource Shortage.

\subsubsection{Security and Privacy}

Security and privacy is also a major factor of the IoV but a way different concern as it is evolving as an entirely a new field altogether. Existing literature [99-100] has revealed that tampering of data at the network may affect lives of the people on roads. Hence, compromising with security shall deeply affect the vehicular environment. Bhardwaj and Khara [101] and Groza and Murvay [102] had highlighted several research challenges for security and privacy which has been focused on the IoV. Philip, et al. [103] had discussed security and privacy in location-based services [104][105] for mobile and vehicular communications. Banani and Goron [106], Hamida and Javed [107] had focused on the issue of signature verification to introduce security and privacy in the IoV applications. Sun [108] had divided attacks into six categories in the STRIDE Spoofing, Tampering, Repudiation, Information disclosure, Denial of service, Escalation of privileges) threat model. Authors in [109110] had reported some counter measures for attacks as discussed in [108]. Sharma and Kaushik [111] had reviewed Evolutionary Algorithms (EAs) from the security and routing point of view with the intention to emphasize the utility of the EAs for solving complex problems where some sort of optimization and searching is required. The research had reported in [111] is limited to EAs only and other nature inspired algorithms has not been well explored in this area of research. However, the use of EAs to ensure security and privacy is a novel approach.

Likewise, Contreras-Castillo et al. [112] had proposed a sevenlayered architecture for IoV to support privacy and security. It is explained that the existing IoV models do not provide (a) security aspects; and (b) interaction with the cars and drivers. The work reported in [113] is the extension of [112] which represented the seven layered IoV architecture with additive security features. Qureshi et al. [114] had supported the claims made in [113] and, had highlighted the importance of integrating cloud computing, big data and security aspects. In another research, Tuyisenge [115] and Ang et al. [116] had also supported seven layered architecture of [112 -113] and had emphasized more on security and privacy. Authors [115-116] had introduced model to addresses the issues related to: (a) security; and (b) provide a better integration of interaction with drivers and cars. But the convention of [115 -116] is limited to the ideas without any validation (the idea was not tested through simulations). Hence, it is hard to believe on their claims.

All in all, researchers had represented their own layered IoV architecture focusing on enabling security and privacy. But it is noted that the inference of all models are the same in terms of their working. It is also observed that most of the proposed architectures are supported 3-layered, 4-layered and 7-layered IoV models as depicted in Figure 7. 
Fig. 7 Different IoV layered

Models: a) 3-layered model b)

4layered model c) 7-layered model

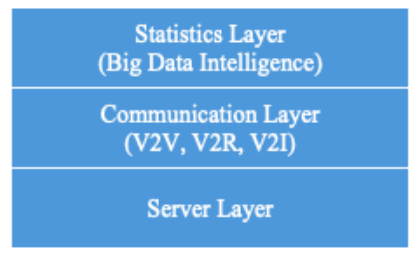

a)

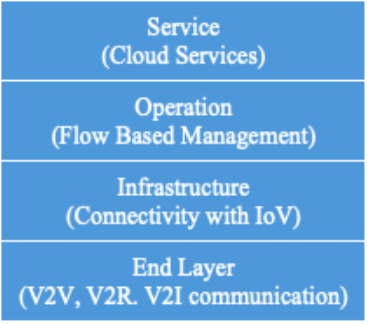

b)

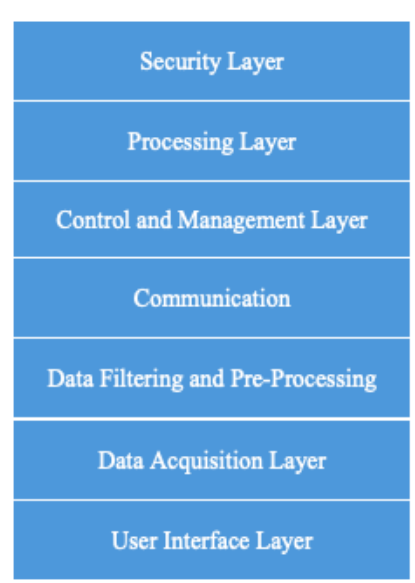

c)
Thus, this study also gave us the future trends in this area like reduction in the anomalies of Intrusion Detection System (IDS), dealing with the 'Big Data', trust and verification of data centers, cross-layer transmission, reliability and delay constraint. Supportably, we infer some of the parameters that affected privacy and security are: Reliability, Availability, Integrity, Secrecy and AuthEntication (RAISE).

\subsection{Theoretical Framework}

Based on the factors and parameters above, there are several models proposed in the literature. Most relevant ones are discussed below, under 6 categorizations: Network QoS, Infrastructure QoS, Performance-Cost, Energy QoS, Big-Data QoS, and Privacy and Security. This section aims at describing the work currently being done by the researchers and highlighting the future scopes for the readers and researchers to suggest potential future directions in the field.

\subsubsection{Network QoS}

Hao et al. [117-118] had presented an empirical study over short-range communications between vehicles and between vehicles and the roadside in a realistic scenario. The V2V and V2R experiments that are conducted mainly measured success ratio, signal attenuation over distance, communication performance in single hop communication. The V2R communications are tested out in the multi-hop communication environment and both single hop and multi-hop results are compared. It is found that communication performance improved in the case of multihop communication scenarios. Future Scope: The future scope of the study is to perform the comprehensive experiment for a broader range considering other factors like packet size, different locations, bandwidth, etc. though the authors had suggested to find other factors that affect communication, which we had already exercised in the previous section.

Dutta et al. [119] had proposed a hierarchical clustering method for multi-hop communication to increase the network lifetime and to ensure proper utilization of network resources. The method is compared to other techniques like n-hop and distributed multi-hop clustering. The results had shown a significant improvement on comparison. They had used neighborhood follow algorithm for the process. Future Scope: However, the efficiency of the algorithm and proposed method can be evaluated using genetic and evolutionary algorithms to prove the obtained results.

Korkmaz et al. [120] had proposed a Controlled Vehicular Internet Access Protocol with QoS (CVIA-QoS) is a solution to multi-hop network routing and spanning MAC functions with infrastructure support. It has executed scheduling decisions and periodic admission control for the packet traffic by establishing fixed gateways along the road. This protocol is considered only for the best-effort traffic scenario by assigning the remaining bandwidth. It has bestowed delay bounded throughput guarantee for soft real-time traffic outstanding the issue of the mobile multi-hop network. This paper also has introduced a routing and channel access technique for Internet connectivity along highways with QoS support in vehicles. The performance of the protocol is evaluated through simulation. Results had shown improvement in throughput capacity and best effort load on realtime throughput. Future Scope: The authors had suggested improving this protocol and accommodating multi-segment reservation strategies and multiple gateways to ensure coordination in the network.

$\mathrm{Du}$ et al. [121] had presented optimized models to characterize the vehicular broadcast capacity in ad-hoc networks. They had basically tried to measure the delivery rate of a message in the VANET setup so as to update with the new message instantly. They had used MitSim Lab to generate the actual traffic flow scenarios and used necessary mathematical tools to estimate the performance of the network in real-time cases that is sure to make a difference in the travel decision making and since this information is necessary for the IOV connected users, the findings will make the knowledge and findings richer in this area. Their results had shown that the performance of VANET is deeply affected by the traffic flow characteristics like traffic speed, density, number of lanes, SINR threshold and transmission range. Future Scope: the findings and models can be tested on the real cases for the analysis of the efficiency of the proposed optimized models in the present scenarios.

Lv et al. [122] had proposed a novel solution called SWIMMING i.e. Seamless and Efficient Wi-Fi-based Internet 
Access for Moving Vehicles. This has an answer to problems like frequent handoffs [123] between access points. This worked in a group unicast manner in uplink which increases reliability and efficient utilization of the channel. In the downlink phase, this approach operates in two stages. This approach has overcome the problems of grey-zone behavior, weak temporal and spatial correlation. The authors had also proposed verification of the ACK detection scheme for the first time and tested it in a real scenario. Also, the probability of successful transmissions is found more in this approach which keeps link bit rate at higher levels. The approach had proved to be superior to the other schemes due to low packet loss rate than other schemes. Future Scope: The authors had claimed to gain higher throughputs in case of multi-radio multi-channel deployment, which is to be tested yet.

Zhang and Liu [124] had given a concept of Code Aided Retransmission based Error Recovery (CARER) scheme to improve the reliability of message broadcast in IoV. This scheme has introduced RTB/CTB (Request to Broadcast/ Clear to Broadcast) handshake to outstand the problem of hidden nodes and high packet collision rate. The results had shown $10 \%$ performance gain using CARER scheme in terms of reduced probability of data transmission collision as the result of the use of network coding technology. Future Scope: The future scope is to work on the end-toend QoS performance for the proposed scheme. Like, Eze et al. [126] had worked on his earlier contributions [125] and had used its proposed CARER scheme to guarantee the reliable and timely broadcast of safety messages [127] for channel access in network communication. The authors had also developed a rebroadcasting metric to avert broadcast storm problems with the use of RTB/CTB packet in broadcast transmission to productively choose a vehicle to rebroadcast the encoded message which ensured reliable delivery and timely broadcast of safety messages. Future Work: The future scope is to work on short road segments with intersections in urban environments with the application of CARER protocol and practical scenarios.

Jin et al. [128] had proposed a remote wireless transparent Construction Vehicles Data Transmission Protocol (CVDTP). The authors had explained the issues of TCP and UDP in regard to remote wireless network transmission and proposed their own protocol to overcome the issues. CVDTP has advanced the technique of window coloration. The field test and the reasonable application on the Internet of concrete pump trucks had shown that CVDTP could bear the cost of QoS information transmission on the remote system portrayed by frequent disconnection and low bandwidth. The test outcomes likewise had demonstrated that CVDTP did not require the help of routing nodes in remote systems and therefore it is effective to be executed. Future scope: Bit error rate is one of the prime concerns of loss of data. The CVDTP only worked on checksum mechanism which can be enhanced to comprehend the specific location of bit error and preserve the existing frame data to the maximum extent.

Tropea and Santamaria [129] had tried to optimize the vehicular traffic with the aim of reduced $\mathrm{CO}_{2}$ emissions and re-routing the traffic to less congested roads. The authors had presented Congestion Avoidance in Vehicular
Environments (CAVE) algorithm based on a weight-oriented graph for this purpose that collected the data from the vehicle to roadside units (RSUs). Also, the re-routing has ensured less residence time on vehicles which is reducing fuel consumption [130], emissions, road congestion and travel time reduction.

He et al. [131] had proposed a new strategy for load balancing for cloud computing using SDN in IoV. The authors had addressed the problems of processing latency, less mobility, and location support by integrating the fog computing strategy with SDN. The had proposed algorithm is SDN based on Modified Constrained Optimization Particle Swarm Optimization (SDCFN). The authors had proposed the theoretical model of SDCFN, then algorithm and further presented the numerical results. Latency performance of bandwidth is evaluated when the task load is uploaded over the cloud and results indicated the use of SDCFN for latency-sensitive services when compared with the cloud. Future Scope: The scope lies in studying more load balancing algorithms that can be used in SDCFN and more parameters other than latency can be considered to evaluate the algorithm performance.

Fabian et al. [132] had aimed at improvising their previous work [133] using SDN model by proposing a new IoV architecture based on programmable Objective Function (OF). They had used a combination metric of delay, ETX, residual energy and velocity of the node to compute rank. The results are compared to OFO and MRHOF standard OF. Improvement in throughput and reduction in energy consumption, end-to-end delay and packet loss could be seen evidently. Future Scope: The solution is found to be less effective in the cases of low mobility and low density areas. Hence, researchers can aim to work at improving the proposed technique for such scenarios.

Huang et al. [134] had proposed a resource allocation scheme for all the cars in the SDN to maximize the Quality of Experience (QoE). They had evaluated the results and found their approach to be efficient with low computational complexity. Future Work: The scope is to consider the handoff problem and channel fading issues in future work for the improvement of this proposed work.

Yuan et al. [135] had given the Space and Time Constrained Data Offloading (STCDO) scheme to improve the overload status of vehicular networks while satisfying spatiotemporal constraints. Future Work: The scope is to improve this scheme by taking advantage of collaboration among subscribers of networks and thus improving the offloading seeds strategy in vehicular networks.

Davydov and Bezzateev [136] had proposed two blockchain based accident detection model to improve detection of law violation by the vehicles. They had proposed a novel strategy called offline detection which targeted unseen accidents on roads and ensures maximum protection to victims. Future Scope: Researchers can target working on the security and privacy aspects of the method with the use of techniques like cryptography mechanisms, secure blockchain protocols, etc.

Other authors like Chang et al. [137], Kunarak and Seleesathira [138], Guerrero-Ibáñez et al. [139], GuerreroIbanez et al. [140], Bitam, et al. [141] had worked on determining the apt. metric to improve network QoS. Future Scope: The creators need to stretch out their outcomes to a setting which contains numerous needs of clients, and, in such a setting, a couple of clients in the higher need may had more thorough QoS prerequisites than low-need clients. Likewise, 
they might want to explore how to structure QoSenhancement arrangements under a setting when clients are non-helpful and every client may dismiss the designation of transmit control with a specific likelihood.

De la Iglesia et al. [142] had proposed an improved calculation for guaranteeing a robust co-existence framework for clients by allocating resources among V $2 \alpha$ clusters with use of non-V2 $\alpha$ User Equipment (UE) on the matching bandwidth. Consequently, this paper has presented an underlay RRM (Radio Resource Management) strategy which is equipped for enhancing cellular spectral productivity while having a negligible effect on cell interchanges and guaranteeing the distinctive QoS of ITS applications. Simulation had shown that their proposed calculation in comparison with the other two RRM techniques, demonstrated the promising spectral proficiency execution of the proposed RRM technique. Future Scope: The researchers may work to get better at the energy efficiency results obtained during the study by designing a more precise control transmission job, which shall decrease the interference generated.

Chen et al. [143] had proposed two models considering the issues of jitter, buffering, throughput and delays while streaming video applications in maintaining QoS requirements in the network. An optimal routing approach is proposed for the selection of paths to reduce latency in video streaming. Numerical simulations are conducted using Matlab. The calculations are done on average onehop coverage, length of a routing path and experienced latency. Simulation results had reflected that the proposed model has elevated precision under diverse configurations. Future Scope: The researchers may work to decrease the estimation error. Also, new scenarios can be considered with more factors like traffic rules, traffic signals, overpasses, etc. that cause delay. The authors also aim to work on the routing overhead and efficiency tradeoff for the proposed algorithm.

Kumar et al. [144] had proposed an efficient traffic control system for IoV and proposed fuzzy logic-based traffic intensifies calculation function to model heavy traffic. The experimental results for the performance had shown that average travel time and average waiting time with respect to traffic density is better in case of the proposed algorithm when compared with the rest. Future Scope: The work on the continuous health monitoring system based on the proposed IoV based traffic management method can be taken up as future work.

Alzamzami and Mahgoub [145] had developed a new geographic routing protocol based on fuzzy logic systems named Fuzzy Logic based Directional Geographic Routing (FL-DGR) that help in analyzing and coordinating contradicting metrics. The results had shown a better performance than Directional Greedy Forwarding using Expected Transmission Count (DGF-ETX), Adaptive Fuzzy Multiple Attribute Decision Routing (AFMADR), Stability and Reliability aware Routing (SRR) and CrossLayer optimized Weight Position-based Routing (CLWPR) in terms of packet delivery rate, total network throughput and end to end packet delay. Future Scope: An adaptive HELLO protocol that changes generation rate according to the density of vehicles and mobility to reduce network congestion.

$\mathrm{Wu}$ et al. [146] had given an optimization scheme for deployment in multimedia edge clouds. This QoS driven IoVs application had reflected optimal results during simulation supporting different QoS requirements in CDN (Content Delivery Network) multimedia edge cloud environment. Future Work: Authors aim to improve the QoS model by adding more data and scaling it by considering more metric parameters.

Venkatramana et al. [147] had proposed a connectivity aware intersection based shortest path routing protocol (CISRP) for urban setup. The results had shown that the average hop count, delivery ratio, latency and routing overhead is less in CISRP when compared with other protocols (Greedy Perimeter Stateless Routing (GPSR), E-GyTAR, TFOR, segment vehicle, Link quality and degree of connectivity based Geographic Distance Routing (SLD-GEDIR)). Future Scope: However, the protocol can be tested in more practical scenarios with variations in the density and topology of the network.

Zhang et al. [148] had proposed a genetic algorithm based on QoS perception routing protocol to guarantee QoS and improvement in the packet transmission and broken links between vehicles in VANET. The numerical results had shown that the proposed algorithm outperformed the intersection-based routing and connectivity aware routing protocols on the grounds of packet delivery rate and transmission delay. Future Scope: The authors had proposed that the suggested scheme is complicated to code using genetic algorithms and slow in searching operation. So, the researchers can work on optimizing the speed by combining the bionic heuristic approach with genetic algorithm in this approach.

Zhou et al. [149] had addressed the issue of content dissemination in vehicular networks and aim to solve it using an edge caching scheme in RSUs. The authors had analyzed the vehicular content requests made by the users using content access pattern, traffic density and position of the vehicle and then, determine how to distribute the information using their proposed cross-entropy based dynamic content caching scheme. The results had shown improvement in terms of performance with additional overhead that needed to be improved. Future Work: The scope is in simulating the algorithm considering different locations and RSUs with different network operators and pricing model of cooperation among RSUs to distribute contents to vehicles.

Mouhcine et al. [150] had proposed an efficient strategy to reduce traffic congestion using VANET schemes with a distributed swarm intelligence optimization technique. The results show that the total time is taken and total distance traveled comes out to be less than the other existing shortest path techniques. The simulation results had shown that the proposed schemes improved network performance in highway and urban environments with different conditions of vehicle density. Future Work: It can be implemented as a Contract Network Protocol (CNP) to improve the effectiveness of the proposed algorithm. Also, scalability and accessibility for the users can also be improved for better credibility.

Philip et al. [151] had worked on the concept of delay to reach near-optimal solutions to reduce delay and had proposed a consensus-based gradient descent and constant step-size algorithm. Future scope: Development of finite time distributed algorithms for the generalized constrained problem in distributed optimization as future work. 
Sherazi et al. [152] had proposed heterogeneous network architecture that supported multiple wireless interfaces. They had used the Best Interface Selection (BIS) algorithm to ensure reliable communication and promised efficient data forwarding in V2I communication. Future Work: Researchers can aim to work on analyzing the operational cost and capital investment in deploying such approaches.

\subsubsection{Infrastructure QoS}

Wang and Lien [153] had given a statistical approach for automatic vehicle detection using local features. They had suggested the use of joint probability of positions and local probability to model the vehicle. They had proposed four solutions namely: first, all sub-regions to be projected without position data into single eigenspace, second, all sub-regions to be projected with position data into a single eigenspace, third, each sub-regions to be projected with position data into corresponding eigenspace, fourth, each sub-regions to be projected with residual independent basis position data into corresponding eigenspace) and had compared all the four solutions based on their testing image dataset. This approach has reduced the alignment error by incorporating geometric distortions of vehicle appearance. Future Scope: This study is conducted with keeping an on front view vehicle image dataset. The future study can introduce a side view of vehicle data with different viewing angles to analyze a broader scope.

Uppoor and Fiore [154] had proposed the microscopic and macroscopic features of pervasive vehicular access. They had tried to investigate the prime metrics for cellular Radio Access Networks (RAN) deployment using the realistic road traffic datasets. The results had shown that the cell residence time did not vary significantly since vehicular access generates significantly low residence times. This finding with high pervasive vehicular access load will make the limiting of handover cost risks complex. Future Scope: The analysis is constrained to one urban scenario and can be extended to upcoming vehicular load in other areas. The findings can be made more precise by working with the actual data that are not publicly available due to security issues.

Martínez-Vidal et al. [155] had measured QoS in remote system engineering of the aeronautical systems. A distinctive approach for the system proposed is to blend the idea of Delay Tolerant Networking (DTN) through the contacts, together with direct satellite access in a set number of the hubs. Acquired outcomes had shown critical enhancements in every single metric pointer with respect to QoS, not generally reachable on the field of DTNs. Future Scope: The presentation of needed dimensions of system traffic is limiting the quantity of satellites and in this way the design cost. Additionally, the thought of twoway correspondences is to perform ground-to air interchanges. This kind of correspondence represents a few nontrivial challenges with respect to gateway determination, routing, and traffic load balance [156][157].

\subsubsection{Performance-Cost}

Lu et al. [158] had examined a performance-cost tradeoff which deploys wireless access infrastructure that offers a downlink data pipe to all vehicles in the vehicular network. They had estimated the lower bound and upper bound of downlink capacity for each infrastructure. They had also conducted a case study and deployed Capital Expenditures (CAPEX) and Operational Expenditures (OPEX) to examine performance-cost tradeoff. The comparison of deployment scales had shown that more Remote Access Point (RAPs) will be required to improve downlink throughput provided the network operates in infrastructure mode which is less than the amount required in hybrid mode. The choice of cost-effective infrastructure should be made depending on the demand for vehicles. The authors had suggested that RAPs can be a good choice in case of high-speed data pipe for vehicles as a costeffective solution. Future Scope: Researchers can propose a model to validate this study by comprehensive simulation and studying its effect on network operation and design.

Salahuddin et al. [159] had proposed a new approach called RSU cloud which is actually a vehicular cloud in IoV. This cloud had comprised conventional and specialized RSUs that use SDN [146] in Mininet to dynamically commence, replicate and moderate services. The aim is to decrease the number of service instances or RSU cloud infrastructure in each configuration for a provided average demand, limit the infrastructure delay and minimize bandwidth resources that weaken the network performance and QoS. Use of the heuristic method of reinforcement learning and Markov Decision Process (MDP) to select Pareto Optimal Frontier (POF) solution is made to decrease the cost of the network for a long span. However, the authors had not compared their joint optimization approach with delay optimization in a purist approach. Also, the results had shown great improvements in infrastructure delay and reconfiguration cost in contrast to purist service installations. Future Scope: To emphasize on working at the load balancing technique to reduce control plane modifications and utilize the mobile OBU resources.

Ligo et al. [160] had said that vehicular mesh networks can provide internet connectivity using DSRC in urban areas in the most cost-effective manner rather than intensifying the competence of cellular networks and had tried to identify such scenarios using their simulation model and dataset from a real vehicular network of Portugal. The model had analyzed the internet load that can be delegated to vehicular networks which in usual cases is boring by cellular networks. They further guesstimate the remuneration of cost savings for this setup in both cases. However, they had claimed that the DSRC-related costs should be acquired to ensure that safety is enhanced. They had also claimed that if DSRC enabled vehicles became mandatory, the results will be appreciably better than the outstanding costs (costs of roadside infrastructure). Future scope: To work over fidelity in traffic per vehicle and the dynamics of penetration, traffic and costs over time.

Wang et al. [161] had addressed the issue of cost and space with biased results in case of manually designed metrics in cluster vehicle trajectory. Hence, they had proposed a dynamic approach for vehicle trajectory clustering using machine learning method like k-means, gaussian mixture model and kmedoids. They had evaluated the performance of their proposed method using real dataset. Their results had shown better cost performance tradeoff than the other baseline methods. Future Scope: Authors aim to explore a k-near neighbor based on IoV to cluster vehicle trajectory in future. Also, other methods can also be considered for comparison that make use of these 
clustering techniques to justify their results.

\subsubsection{Energy QoS}

Kamgueu et al. [162] had conferred the realization of routing metric considering Energy Transmission Exchange (ETX), delay and energy as its parameters using Mamdani model of fuzzy logic [163-165] to devise RPL objective function. The simulation was done using the Contiki operating system and deployment was conducted on a realworld indoor WSN. The results for this setup had shown improvements as compared to the RPL protocol. However, the packet loss ratio over time at the sink node and a number of parent changes per hour is higher than the native RPL protocol. Future Scope: To compare this proposed metric approach with other metrics. and work on the QoS requirements since the network can generate various traffic flows with variant components like end-to-end delay, transmission accuracy, etc.

Lamaazi and Benamar [166], Lamaazi et al. [167] had considered fuzzy logic to improve energy efficiency. In their next work they had proposed a new objective function to choose the best parent using Routing Protocol for Low Power and Lossy Networks (RPL). Their aim to improve energy efficiency is then compared with the standard approaches and their previous work. The simulation results had shown improvement in the energy consumption. However, the instability of links and increased network lifetime became a concern due to multiple times selection of parents is performed as per the proposed algorithm. Future Work: Researchers can work on improving the network lifetime, unstable links and other parameters can be considered like residual energy and convergence time which are currently an issue in the proposed method.

Priyan and Devi [168] had proposed an energy efficient node selection algorithm based on the performance index and the Random Waypoint Mobility Model (RWMM). The performance metric is evaluated over the medical capacity of the mobile ambulance, no of patients using the ambulance and the Euclidean distance from the nearby mobile ambulance. RWMM model is used to simulate the IoT based healthcare monitoring system. Packet Delivery Fraction (PDF), Average End to End Delay (AED) and Normalized Routing Load (NRL) are evaluated with respect to node density and connection rate to evaluate the performance of the proposed algorithm. The results had shown the proposed algorithm outstanding in all the three cases of PDF, AED, and NRL when compared with the other algorithms. Thus, the authors had tried to improve the energy efficiency of the node selection.

Mukherjee and De [169] had proposed a Channel aware Dynamic Window Protocol for energy efficient communications (cDIP). The had proposed communication strategy depends on the rate of variation and not on the fading distribution of the channel. The conventional Automatic Repeat Request (ARQ) is modified for shortrange wireless communications. The numerical results show a gain of $40 \%$ in data throughput and $41 \%$ in energy efficiency as compared to existing schemes. Future Scope: Several parameters of energy metrics remain undiscovered with respect to the implementation of MAC and network protocols.
Zhang et al. [170] had focused on the necessity to improve battery depletion in Electronic Vehicles (EV) and suggested dynamic charging piles using recommendation method. They had proposed the method based on three mechanisms: data acquisition, judgment of the surrounding environment and user's intention to charge or not and finally, if interested dynamic charging mechanism is used to evaluate indexes for the proposed scheme. This experiment had shown that the equilibrium utilization rate of charging piles can be improved. Future Scope: Researchers aim at improvising the recommendation algorithm. However, other factors like residual energy, charging capacity, battery life can also be taken into consideration while evaluating the performance.

\subsubsection{Big-Data QoS}

Chiti et al. [171] had discussed user association techniques to optimize data distribution in IoV. The authors had proposed two matching based user association techniques. One is clustering based and it is modeled as Hospital Resident (HR) matching game and second provides an equal and independent relationship between vehicles, distinguished from coordinated relation in clustering mode. Irving's Stable Fixture (ISF) algorithm is used to evaluate stable matching between vehicles if one exists. The authors had proposed two matching based user association techniques. The had proposed techniques are compared with heuristic algorithms on various grounds like network partition, connectivity, and overall data exchanged. The results had shown the highest connectivity degree in Stable Fixtures (SF) especially in low to medium level congestion, SF outperformed in case of a number of partitions with larger communities. SF had shown exceptional payoff enhancement in case of increased vehicular density while maximizing the information sharing. The SF model is flexible and scalable. Chaqfeh et al. [172] expressed the need for data collection and processing of vehicular clouds for ITS. They had tried to show that a lesser number of vehicles is sufficient to gather reliable information for understanding the vehicular network.

Amadeo et al. [173] had presented the problems of NDN schemes of not being able to prioritize traffic treatment based on the requirements of heterogeneity in IOV. So, the authors had proposed a holistic NDN approach to ensure right prioritization based on the demands of encoded data traffic in NDN data names and shape NDN forwarding decisions dynamically. The authors had named it prioritized-NDN (p-NDN). This study has first selected outgoing gateway for NDN packets and then up righted the timing of broadcast transmissions over the 802.11 Outside the Content of a Basic Service Set interface. The comparison is carried out between the single face NDN and multiple faces NDN approach for non-prioritized and prioritized schemes both. The simulation results had shown that the proposed technique reflects enhancements in accomplishing high delivery of packets with reduced latency at the cost of slightly higher traffic [174-176] load overhead. Future Work: To develop better multi-face forwarding algorithms with better data sharing at low latency and reduced overhead. The authors also aim to work on the D2D technology for V2V communications when it gets fit for use. Also, the number of requests received can be dynamically measured at the interfaces and mechanism to improve performance by allowing prompt switching from one interface to another can also be some of the future goals.

Cho and Kim [177] had informed that so far genuine 
information-driven investigation, e.g., using machinelearning, has utilized information from sensors, for example, GPS and accelerometer. They had performed preprocessing ventures to clean and refine the raw information, consequently converting the outcomes into csv documents, which are good with a wide assortment of analysis devices. They had additionally given example cases to exhibit strategies for recognizing anomalous driving examples, for example, moving over a hindrance. Future Scope: This dataset will be helpful for specialists refining their investigations of vehicle developments. This work will be discharged as an open dataset and be helpful for specialists in the field to perform vehicle development examination.

Xie et al. [178] had proposed STM32-based vehicle data acquisition approach for IoV to improve the feasibility and effectiveness of DAS (Data Acquisition Systems). Yanting et al. [179] had expressed that the vehicle state is essential to predict the vehicle's position, acceleration and velocity. The authors had proposed called Driver Behavior Decision Tree (DBDT), the use of a decision tree as a fast situation judgment and consistent method to predict the vehicle state. Some trajectory prediction methods are proposed that are based on lane changing recognition. The experiments are performed in real environments base on Lankershim Boulevard Dataset of the Next Generation Simulation (NGSIM) simulator. The accuracy of position prediction, velocity prediction and acceleration prediction for DBDT is compared with other position prediction strategies like Constant Yaw Rate and Acceleration (CYRA), Decision Tree (DT) and Self-Selection Decision Tree (SDT). Future Scope: Social Internet of Vehicles is also coming up with different characteristics and complicated circumstances of the data analysis. Researchers can aim to work over those problems in the near future.

Fisher et al. [180] had added to the plan and actualized a versatile explanation framework that can give an exhaustive arrangement of picture names for extensive scale driving datasets. A driving dataset is encouraged by the proposed annotation tool that includes over $100 \mathrm{~K}$ recordings with different annotations like object bounding boxes, image-level tagging, drivable areas, full-frame instance segmentation, and lane markings. The authors also divide the drivable area into the directly and alternatively drivable area and propose a new approach for road segmentation. The dataset has geographic, ecological, and climate decent variety, which is helpful for preparing models so they are less inclined to be amazed by new conditions. The dataset isn't open access. This dataset accompanies thorough explanations that are fundamental for a total driving framework. In addition, tests demonstrated this new dataset is more testing and more complete than existing ones and can fill in as a decent benchmark for area adoption because of its assorted variety. Future Scope: This dataset can be explored by the researchers using various deep learning and mining techniques to extract the relevant information as per the studies.

\subsubsection{Privacy and Security}

Smitha et al. [181] had raised the concern of security of data in the vehicular network in their article and so proposed an optimized adaptive algorithm for authentication of safety messages in VANET or connected vehicles. Yang et al. [182] had worked on the efficiency and security of IoV and had studied anomaly detection in internet-connected vehicles. They had presented a trust-based anomaly detection scheme with affinity propagation. The proposed technique had detected abnormal vehicles, had established communication with each other and finally, had congregated to trustworthy Cluster Heads $(\mathrm{CH})$. The scheme is improved with sparse RSU-central reputation provision scheme where Central Arbitrator (CA) collects sparse RSUs and distributed supervising technique to ensure fairness and robustness in detecting process. A reputation system is a setup to analyze the global reputation from accumulated data. The had proposed scheme could accomplish low detection failure rate below $1 \%$ and filter abnormal vehicles. Future scope: To work on the security of RSUs and in-vehicular systems deploying local and mobile CA using cloud computing schemes, reducing overhead in anomaly detection and improving mutual trust evaluation.

Makarfi et al. [183] had worked on improving the security at the physical layer. They had used double Rayleigh fading channel to capture the mobility effect within the channel of communication. The results had shown that the distance of nodes and eavesdropper node played an important role in the analysis. Future Scope: Although, PLS in itself is a new and very less explored area as of now, yet the researchers can work on evaluating the Physical layer security framework on grounds other than privacy and secrecy also.

Kang et al. [184] had worked on the issues of location privacy in Cloud Enabled IoV (CE-IoV) technology. They had presented two unexplored Virtual Machine (VM) mapping attacks and proposed a pseudonym-changing synchronization scheme and VM identifier replacement scheme to ensure location privacy. The simulation results had shown that QoP is achieved best in the third scenario done using PCSS (Pseudonyms Changing Synchronization Scheme) in small and large observation regions both. Their proposed schemes prove to be efficient in providing privacy preservation with high QoP for safe driving.

Joy and Gerla [185] had portrayed a few vehicular applications that influence V2V and V2I. They address these issues and all the more explicitly center on the need to ensure area security to portable clients. They had assessed the anonymized Local Privacy system over a real dataset. They had relegated virtual characters to every vehicle and had contrasted the Anonymized Local Privacy component with the ground truth information over a 24-hour time span with a confidence interval of $95 \%$. They had presented the idea of Haystack Privacy, which qualifies in protection quality as more information proprietors take interest yet looks after precision. They had shown that information can be privately gathered into a typical open information vehicular database to be shared among various colleagues using the Haystack Privacy instrument.

Pokhrel and Choi [186] had proposed the concept of federated learning framework to enhance the IoV performance. They had used analytical modelling to stabilize the data flow over Wi-Fi networks by using TCP Cubic streams. Their mathematical analysis had shown comparative results with the existing Wi-Fi setup. The proposed framework can also provide privacy without the loss of performance. Future Scope: However, the algorithm needs further investigation and experimentation in 
real scenarios to know its actual working and efficiency.

$\mathrm{Xu}$ et al. [187] had communicated that different authentication schemes for multi-authentication models are proposed and connected to numerous zones, however less in the IoV. The authors, therefore, had proposed another Mutual Authentication and the Key Agreement Scheme (LEANDER) under multi-server design for Long-Term development for Vehicle (LTE-V) to organize for unsure driving conditions. In this plan, the elliptic curve is utilized to diminish the computational complexity and a progressively compact authentication strategy is developed. Random anonymity has bolstered multi-server for two-way validation and key understanding, to successfully ensure the security of the vehicle. In addition, it has utilized a BAN rationale to demonstrate and break down the viability of this plan. The LEANDER utilizing ECC had enhanced and guaranteed the verification procedure. When contrasted with existing plans, the LEANDER can incredibly decrease the computational expense and develop straightforward verification techniques. The execution investigation results had demonstrated that the proposed authentication scheme is compelling and more secure than other schemes.

Ahmed et al. [188] had proposed a light weight key agreement protocol for SIoV. They had used cryptographic operations like hash, exclusive OR and validated the model using formal security model. Their results had shown that the computation cost and storage for the proposed approach is lesser than the base line techniques. Future Scope: The method can be improvised by testing it against various network attacks.

\section{Research Gaps and their analysis}

Based on the comprehensive study of the literature, we propose the present challenges in IoV, their future directions and real-time applications in a nutshell. IoV and SIoV plan to coordinate numerous vehicles and clients utilizing heterogeneous IoT advances. The advantages of IoV [189] empower the arrangement of administrations to plenty of utilization to give a progressively dependable, productive and reasonable framework. Notwithstanding, numerous contemporary issues are repressing the further advancement and adjustment of the IoV idea like:

\subsection{Big Data Pre-Processing}

The transport of enormous information, particularly video, over the IoV, is disturbing the system. Enhancement of information preparation must upgrade the execution of IoV innovation. It is anticipated that one-fifth vehicles on the street will have web association and worldwide vehicular traffic to achieve 300,000 Exabyte by 2022 . Such sort of vehicular information blast needs to process before transmission over the system or else the IoV innovation will crash steeply.

The pre-processing of big data will prove to be very effective as the rest of data analysis like data dissemination, data transmission without latency, etc. is based on the effective pre-processing of data. But since $\mathrm{IoV}$ is an emerging field, a smaller number of datasets as per interests of the researchers are available. Moreover, due to security reasons, it is difficult to gain access to the actual data at the vehicular cloud thus; similar experimental setups are conducted to test the effectiveness of the proposed solutions. This will certainly help users to make more informed and in time decisions. Also, it will benefit the transportation industry in different ways like traffic optimization, cyber security and intelligence, weather forecasting, etc.

\subsection{Standardization}

No such fixed standards are accessible to handle issues, for example, interoperability, kinds of equipment and programming, utilization of frequencies, conventions and correspondence innovation. The standards available are basically for the IoT execution. There are no specific standards for the IoV. Consequently, the researchers are proving operational and dedicated standards for IoV development.

The plus point about no rigid standards is the flexibility of development but at the same time the lack of them makes the development aimless wandering because every other innovation is made with the authors' perspectives and it is not practically possible to deploy the standards as per everyone's proposal. Standardization across the IoV landscape will reduce the gap of interoperability among protocols syntax, physical communications systems, data semantics and network deployment.

\subsection{Adaptability}

A significant component of $\mathrm{IoV}$ is mingling and sharing of data, however, data sharing probably won't claim every one of the clients in a few nations because of some hindering elements, for example, culture, protection, trust, security, obscurity, and robotization.

The adaptability of the IoV technology begins with the development of the IoV infrastructure and the adaptability of IoV will require a hefty cost involvement. So, this will for sure enhance the standards of living for the nations but at the same time will need a huge cost involvement for the infrastructure setup and the adaptation of the IoV technologies. Adopting IoV services standardly will lead to the evolution of smart cities, smart retail, emergency care, smart agriculture, transportation, industry management and many more domains.

\subsection{Network Connectivity and Scalability}

The execution of IoV is constrained by the adaptability of the IoT foundation to deal with the dynamic rate of traffic in various zones. The absence of coordination and correspondence is the greatest test to IoV execution. The network is the foundation of the concept of the IoV. With the increase in the traffic density, network load, big data generation, it is mandatory to establish strong internet connectivity with low latency, low/distributed network load, high transmission speed and capability to scale the increasing traffic and data density.

The prospect of establishing high-speed internet connectivity will provide effective deployment of IoV and realize the concept of smart cities. At the same time, the spectrum shortage and unavailability of resources is a major challenge to bestow internet connectivity with full efficiency and capacity. Also, the network transmission, network QoS, network routing protocols and interoperability in IoV are not fully functional and deployed. In terms of applications, IoV connectivity will bring digital 
transformation. By leveraging data like speed, location, traffic, temperature etc. with a stable network, users will be able to predict forthcoming situations like traffic assessment, car parking, road safety, etc.

\subsection{Infrastructure}

Numerous nations are building up cutting-edge vehicles at a fast pace; in any case, they are as yet ailing in the fundamental foundation with regards to the most recent innovative ideas, for example, SIoV. Also, no such predetermined and rigid infrastructure is available in the context of IoV execution. The above structure has made it clear to understand the need to develop an infrastructure for the application of IoV technology.

The development and adaptation of infrastructure will make the deployment of IoV for the nations very bright. It will also facilitate the researchers of the nations to design an experiment and analyze the results in a real environment. The problem arises due to bulky cost involvement and it will be an issue for the conservative areas to build an infrastructure. The applications are well listed as smart homes, smart cities, smart transportation, inventory management, fleet management etc.

\subsection{Energy Efficiency}

The improvement of the IoV faces difficulties like enhancing traffic proficiency, eco-friendliness, fuel efficiency and upgrading traffic security to give individuals progressively advantageous and agreeable encounters of driving. The energy efficiency at nodes, edge devices, routers in the networks, sensors, etc. is of paramount importance. The study has built up the reasons necessary to make energy efficiency significant to implement IoV technology.

The improvement in energy efficiency will solve the other interlinked features of IoV like network connectivity lifespan, non-depletion of energy at the routers, edge devices and other devices employed to ensure IoV deployment. The energy efficient IoV applications like smart tracking, smart lighting system, traffic monitoring etc. can be very significant for future advancement in vehicular networks.

\subsection{Privacy and Security}

Clients' security is critical in IoV, as they should be urged to receive new advancements, especially in those nations with a general traditionalist culture. Be it IoT, IoV, any fully developed and functional technologies or the developing technologies, it is important to ensure security and privacy in all areas.

There are many authentication schemes proposed by the researchers to ensure privacy and security but since not all issues and loopholes in the network could be detected. It is because of not fully functional network connectivity implementation, so detection of loopholes in the network from where it could result in active or passive attacks is needed and then authentication, integrity, and availability of vehicular information through the cloud and other means to users shall be helpful. Unleashing the need of privacy and security are the applications that can help in network threats assessment, home invasion detection and other cyber-crime prediction and intelligence.

\section{Summary and Analysis of Reviewed Literature}

The Internet of Vehicles will help lighten traffic, decreasing the 250 hours the driver spends in the driver's seat a year. It will likewise eliminate crashes, conceivably up to 80 percent by 2035 , in light of the fact that, as Kevin Curran, IEEE Senior member and a professor of cyber security at Ulster University say "self-driving cars don't get distracted as much." Sukanya Mandal, IEEE member (India) say: "It's always been challenging to keep track of the vehicle that is out for logistical delivery. In my opinion, the Internet of Vehicles plays a significant role in the safe delivery of goods as it enables realtime tracking of shipments, warehouse-capacity optimization, predictive asset maintenance, route optimization, and improved last-mile delivery." The literature study is extremely comprehensive to understand the work in the field of IoV till date but as a researcher, it is of paramount importance to know the available tools and simulators to carry the work forward in the field. Special consideration has been taken in this section as no reviewed literature gives a summary of the simulators used to date in the field of IoV in Table 6.

Table 6 List of Simulators used in IoV for V2X testing and simulations

\begin{tabular}{lllllll}
\hline $\begin{array}{l}\text { S. } \\
\text { No. }\end{array}$ & Simulators & Source & Language & $\begin{array}{l}\text { Mobility/ } \\
\text { Scope }\end{array}$ & $\begin{array}{l}\text { Platform } \\
\text { Supported }\end{array}$ & Used by Researchers for simulations \\
\hline $\mathbf{1}$ & NS-2 & Open & C/C++ & Microscopic & Windows/Linux & {$[119][122][123]$} \\
$\mathbf{2}$ & NS-3 & Open & C/C++ & Microscopic & Windows/Linux & {$[28][131][146][171]$} \\
$\mathbf{3}$ & CONTIKI & Open & C/C++ & Microscopic & Linux & {$[166][167]$} \\
$\mathbf{4}$ & MITSIM Lab & Open & C/C++ & Microscopic & Linux & {$[117][118]$} \\
$\mathbf{5}$ & MATLAB & Commercial & C/C++ & Microscopic & Windows/Linux & {$[120][134][142][147][149][183][187]$} \\
$\mathbf{6}$ & SUMO & Open & C/C++ & Microscopic & Windows/Linux & {$[28][145][146][148][149]$} \\
& & & & & & {$[152][168][169][133][171]$} \\
$\mathbf{7}$ & VEINS & Open & C/C++ & Microscopic and & Windows/Linux & {$[127][168][169]$} \\
$\mathbf{8}$ & OMNeT++ & Open & C/C++ & Microscopic & Windows/Linux & {$[127][145][168][169][133]$} \\
$\mathbf{9}$ & TransModeler & Commercial & C/C++ & Microscopic & Windows & {$[182]$} \\
$\mathbf{1 0}$ & MiNiNet & Open & Python & Microscopic & Linux & {$[40][157][158]$} \\
$\mathbf{1 1}$ & ONE & Open & Java & Microscopic & Windows/Linux & {$[144]$} \\
$\mathbf{1 2}$ & EstiNet & Commercial & C/C++ & Microscopic and & Linux/Unix & {$[59][151]$} \\
& (NCTUns) & & & Macroscopic & & \\
$\mathbf{1 3}$ & TSIS-CORSIM & Open & C/C++ & Microscopic & Windows & {$[190]$} \\
\hline
\end{tabular}




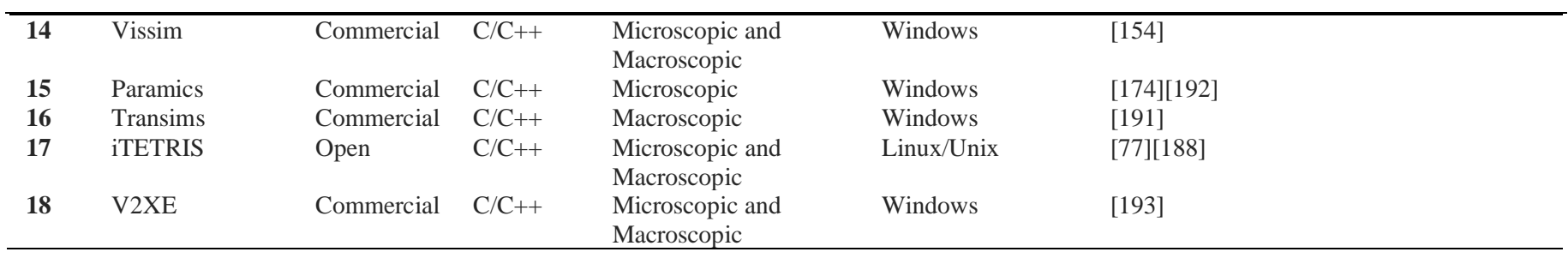

\section{Conclusion and Future Work}

This study presents the diverse definitions of IoV. This paper discusses the evolution of the concept of IoV to present. The study has listed the factors affecting IoV execution. It gives a comprehensive outlook of the proposed methods in the literature which has not been evaluated yet. The advantages and disadvantages of the aforementioned studies have been discussed. Further, the paper gives a detailed overview of the simulation works carried out by different researchers and academicians and lists out the future perspective of those works for the readers and researchers to utilize it as their further studies on IoV. The research gaps are clearly stated in the paper and the gap analysis with the strengths and weaknesses are mentioned that will prove useful for future directions in the field of IoV. Finally, the paper has presented for the first time, the summary and analysis of the reviewed literature with reference to the simulators considered for the related studies in this field. The future work for us will be working on these challenges and to bring advancements in the field of IoV.

We have reviewed the relevant literature and have highlighted the research gaps. Based on this study, we further discuss the potential future directions. Limitations of the study points, towards these future works that can be addressed in the near future:

\subsection{Network QoS}

As we have discussed of Network with QoS, we found data communication is a major concern for the researchers. Data communication in case of single and multi-hop can conduct comprehensive experiments for a broader range like packet size, bandwidth, etc. The algorithms based on routing protocols for the network can be improvised using evolutionary and genetic algorithms. The concept of multiple gateways instead of single gateway at the network border can solve problems of delay and latency in transmissions. Bit rate is a prime concern for loss of data. Most of the proposed algorithms are less efficient for high density and high mobility networks. Novel heuristic approaches can be experimented to resolve such issues of scalability, hard handoff problem, energy efficiency, traffic load-balance, etc.

\subsection{Infrastructure QoS}

Speedy work is going to reform infrastructure for IoV to ensure its full-fledged deployment. However, researchers have faced issues in exploring datasets due to insufficient resources. Creation of primary dataset is possible but it is at same time, time consuming and varies from person to person. So, it opens field for the data miners, researchers and scholars in many ways. Relevant datasets can be made and extracted for others to explore. Existing datasets can be further explored to achieve results. Researchers can aim on exploring other than urban areas also. Road and IoV analysis can be done by considering directions other than front ways to understand the surroundings and flow of adjacent traffic in smart corridors, etc.

\subsection{Performance-Cost}

Since, this field is in its development phase, the resources are not available for all. This brings in the tradeoff between other factors like energy, traffic monitoring, load-balancing, performance, etc. with the cost. In order to subside this tradeoff, more advancement is required to understand how these dependent factors can be exercised and deployed with minimum cost.

\subsection{Energy QoS}

IoT makes use of electronic devices called motes, which contain sensors that helps to communicate with the nearby objects. These motes are used within the IoV setup also for communication. These motes has a battery life which depletes with time depending on the usage. The dependent variables like Expected number of Transmissions (ETX), delay, power consumption, residual energy, latency, etc. play an important role. Researchers targeting to provide QoS in case of energy must focus on reducing these factors to make devices energy efficient.

\subsection{Big-Data QoS}

Big-Data is a huge concern now and so is its pre-processing. Storing and mining of these large chunks of data and extracting relevant information within seconds and then transmitting it to users, is expected for a successful IoV smart corridor. Hence, handling of big-data needs strenuous attention. Researchers can work on suggesting methods, algorithms and technologies that can provide data uploading, forwarding, processing, etc. at a faster convergence rate with a low latency. Various data mining and data extraction techniques can be used acquire relevant data before feeding it to the Vehicular Network Cloud. The preprocessed information at the cloud will surely enhance its capacity to store, transmit at a faster rate and with less delay.

\subsection{Privacy and Security}

Vehicular Mobile Network and cloud computation involves a lot of privacy and security related concerns. They are always 
open to malicious network attacks, infringement, hacking and unethical use of information. Therefore, a strong secure system that can protect the network is mandatory. Researchers can work on providing secure channels for transmission by proposing new and tougher ways of encrypting the data. They can pre-test their algorithms against various network attacks for a better and secure framework. Privacy and secrecy can be provided at the network level also like securing the physical layer, transmission layer, etc. Open challenges like QoP support for pseudonyms-based schemes and improvement in privacy preservation protocols for the communication between vehicles and cloud in a dynamic topology environment with reduced system cost is highly recommended.

\section{References}

1. Stankovic JA (2014) Research Directions for the Internet of Things. IEEE Internet of Things Journal 1(1):3-9, DOI 10.1109/jiot.2014.2312291,

https://dx.doi.org/10.1109/jiot.2014.2312291.

2. Minerva R, Biru A, Rotondi D (2015) Towards a definition of the Internet of Things (IoT). IEEE Internet Initiative 1:1-86.

3. Sun W, Liu J, Zhang H (2017) When Smart Wearables Meet Intelligent Vehicles: Challenges and Future Directions. IEEE Wireless Communications 24(3):58-65, DOI 10.1109/mwc.2017.1600423,

https://dx.doi.org/10.1109/mwc.2017.1600423.

4. Masse RA, Contreras (2019) Application of IoT with haptics interface in the smart manufacturing industry. International Journal of Combinatorial Optimization Problems and Informatics 10:57-70.

5. Jo K, Kim J, Kim D, Jang C, Sunwoo M (2014) Development of Autonomous Car-Part I: Distributed System Architecture and Development Process. IEEE Transactions on Industrial Electronics 61(12):7131-7140, DOI 10. 1109/tie.2014.2321342, URL https://dx.doi.org/10.1109/tie.2014.2321342.

6. Mehar S, Zeadally S, Remy G, Senouci SM (2015) Sustainable Transportation Management System for a Fleet of Electric Vehicles. IEEE Transactions on Intelligent Transportation Systems 16(3):1401-1414, DOI 10.1109/tits.2014. 2367099, URL https://dx.doi.org/10.1109/tits.2014.2367099.

7. S. Zeadally S, Hunt R, Chen YS, Irwin A, Hassan A (2012) Vehicular ad hoc networks (vanets): status, results, and challenges. Telecommunication Systems 50:217-241.

8. Datta S, Kanti (2016) Integrating connected vehicles in Internet of Things ecosystems: Challenges and solutions. IEEE 17th International Symposium on A World of Wireless, Mobile and Multimedia Networks (WoWMoM) IEEE Vehicular Technology Magazine 12:26-35.

9. Wang J, Shao Y, Ge Y, Yu, R (2019) A survey of vehicle to everything (v2x) testing. Sensors 19:334- 334.

10. Kumar R, Dave M (2012) A review of various vanet data dissemination protocols." International Journal of u-ande-service. Science and Technology 5:27-44.

11. Rawat DB, Bista BB, Yan G, Olariu S (2014) Vehicle-to-vehicle connectivity and communication frame- work for vehicular ad-hoc networks. Eighth International Conference on Complex, Intelligent and Software Intensive Systems.

12. Zhao Y, Zhang H, Sun W, Bai Z, Pan C (2014) Performance evaluation of IEEE $802.11 \mathrm{p}$ vehicle to infrastructure communication using off-the-shelf IEEE 802.11 a hardware. 17th Inter- national IEEE Conference on Intelligent Transportation Systems (ITSC).

13. Amodu OA, Othman M (2018) Machine-to-Machine Communication: An Overview of Opportunities. Computer Networks 145:255-276, DOI 10.1016/ j.comnet.2018.09.001, URL https://dx.doi.org/10.1016/j.comnet.2018.09. 001.
14. Hobert L, Festag A, Llatser I, Altomare L, Visintainer F, Kovacs A (2015) Enhancements of V2X communication in support of cooperative autonomous driving. IEEE Communications Magazine 53(12):64-70, DOI 10.1109/ mcom.2015.7355568, URL https://dx.doi.org/10.1109/mcom.2015.7355568.

15. Wang, X., Ning, Z., Hu, X., Wang, L., Guo, L., Hu, B., \& Wu, X. (2019). Future Communications and Energy Management in the Internet of Vehicles: Toward Intelligent Energy-Harvesting. IEEE Wireless Communications, 26(6), 87-93.

16. Mondal A, Mitra S (2020) Security Issues in Vehicular Ad Hoc Networks for Evolution Towards Internet of Vehicles. In: Connected Vehicles in the Internet of Things, Springer, pp 253-307.

17. Belavadi SS, Malik V, Udayakumar T, Srinivas A, Mohan R (2017) $\mathrm{IoV}$ based dynamic batch formation and scheduling technique for driverless vehicles. IEEE Region 10 Symposium (TENSYMP).

18. Borcoci E, Obreja S, Vochin M (2017) Internet of Vehicles Functional Architectures-Comparative Critical Study. The Ninth International Conference on Advances in Future Internet.

19. Kong L, Khan MK, Wu F, Chen G, Zeng P (2017) MillimeterWave Wireless Communications for IoT-Cloud Supported Autonomous Vehicles: Overview, Design, and Challenges. IEEE Communications Magazine 55(1):62-68, DOI 10.1109/mcom.2017.1600422cm, URL https://dx.doi.org/10.1109/mcom.2017.1600422cm 2017.

20. Liu J, Zhang S, Sun W, Shi Y (2017) In-vehicle network attacks and counter- measures: Challenges and future directions. IEEE Network 31(5):50-58.

21. Abbas MT, Muhammad A, Song WC (2020) SD-IoV: SDN enabled routing for internet of vehicles in road-aware approach. Journal of Ambient Intelligence and Humanized Computing 11(3):1265-1280, DOI 10.1007/s12652- 019-01319-w, URL https://dx.doi.org/10.1007/s12652-019-01319-w.

22. Xu W, Snider J, Wei J, Dolan JM (2015) Context-aware tracking of moving objects for distance keeping. 2015 IEEE Intelligent Vehicles Symposium (IV) pp 1380-1385.

23. Tian XY, Liu YH, Wang J, Deng WW, Oh H (2016) Computational Security for Context-Awareness in Vehicular Ad-Hoc Networks. IEEE Access 4:5268- 5279, DOI 10.1109/access.2016.2598155, URL https://dx.doi.org/10.1109/ access.2016.2598155.

24. Khelifi H, Luo S, Nour B, Moungla H, Faheem Y, Hussain R, Ksentini A (2020) Named Data Networking in Vehicular Ad Hoc Networks: State-of-the-Art and Challenges. IEEE Communications Surveys \& Tutorials 22(1):320-351, DOI 10.1109/comst.2019.2894816, URL https://dx.doi.org/10.1109/comst. 2019.2894816.

25. Kerrche CA, Ahmad F, Elhoseny M, Adnane A, Ahmad Z, Nour B (2020) Internet of Vehicles Over Named Data Networking: Current Status and Future Challenges. In: Emerging Technologies for Connected Internet of Vehicles and Intelligent Transportation System Networks, Springer, pp 83-99.

26. Yang F (2014) An overview of internet of vehicles. China Communications 11:1-15.

27. Singh D, Singh M (2015) Internet of vehicles for smart and safe driving. 2015 International Conference on Connected Vehicles and Expo (ICCVE).

28. Jiau, Jiau MK, Huang SC, Hwang JN, Vasilakos AV (2015) Multimedia Services in Cloud-Based Vehicular Networks. IEEE Intelligent Transportation Systems Magazine 7(3):62-79, DOI 10.1109/mits.2015.2417974, URL htps://dx.doi. org/10.1109/mits.2015.2417974

29. M. Amadeo M, Campolo C, Molinaro A (2012) CRoWN: ContentCentric Net- working in Vehicular Ad Hoc Networks. IEEE Communications Letters 16(9):1380-1383, DOI 10.1109/lcomm.2012.072012.120282, URL https://dx.doi.org/10.1109/lcomm.2012.072012.120282.

30. Amadeo M, Campolo C, Molinaro A (2013) Design and analysis of a transport- level solution for content-centric vanets. 2013 IEEE Inter- national Confer- ence on Communications Workshops (ICC) pp 532-537.

31. Senouci O, Aliouat Z, Harous S (2019) A review of routing protocols in internet of vehicles and their challenges. Sensor Review 39(1):5870, DOI 10.1108/sr-08-2017-0168, URL https://dx.doi.org/10.1108/sr-08-2017-0168. 
32. Wan, Shaohua, Gu R, Umer T, Salah K, Xu X (2020) Toward Offloading Internet of Vehicles Applications in 5G Networks. IEEE Transactions on Intelligent Transportation Systems.

33. Maglaras L, Al-Bayatti AH, He Y, Wagner I, Janicke H (2016) Social internet of vehicles for smart cities. Journal of Sensor and Actuator Networks 5:3-3.

34. Siddiqa A, Shah MA, Khattak HA, Akhunzada A, Ali I, Razak ZB, Gani A (2018) Social Internet of Vehicles: Complexity, Adaptivity, Issues and Beyond. IEEE Access 6:62089-62106.

35. Zhang W, Xi X (2016) The innovation and development of Internet of Vehicles. China Communications 13(5):122-127, DOI 10.1109/cc.2016.7489980 https://dx.doi.org/10.1109/cc.2016.7489980.

36. Bechler M, Wolf L, Storz O, Franz WJ (2003) Efficient discovery of Internet gateways in future vehicular communication systems. The 57th IEEE Semiannual Vehicular Technology Conference, 2003 VTC 2003-Spring 2.

37. Hussain R, Abbas F, Son J, Kim S, Oh H (2014) Using public buses as mobile gateways in vehicular clouds. 2014 IEEE International Conference on Consumer Electronics (ICCE) pp 175-176.

38. Jiacheng C, Haibo ZH, Ning Z, Peng Y, Lin G, Sherman SX (2016) Software defined Internet of vehicles: architecture, chal- lenges and solutions. Journal of communications and information networks $1: 14-26$.

39. Kehal M, Zhang ZJ (2018) Social internet of vehicles: an epistemological and systematic perspective. Library Hi Tech 38(1):221-231, DOI 10.1108/Iht-12-2017-0259, URL https://dx.doi.org/10.1108/lht-12-2017-0259.

40. Campolo C, Molinaro A, Iera A (2018) A reference framework for social- enhanced Vehicle-to-Everything communications in 5G scenarios. Computer Networks 143:140-152, DOI 10.1016/j.comnet.2018.07.010, URL https://dx. doi.org/10.1016/j.comnet.2018.07.010.

41. Awang A, Husain K, Kamel N, Aissa S (2017) Routing in Vehicular Ad-hoc Networks: A Survey on Single- and Cross-Layer Design Techniques, and Perspectives. IEEE Access 5:9497-9517, DOI 10.1109/access.2017.2692240, URL https://dx.doi.org/10.1109/access.2017.2692240.

42. Dhall N (2017) A survey on Cross Layer Designs for Routing in Vehicular Ad-hoc Networks.

43. Abbasi I, Khan AS (2018) A Review of Vehicle to Vehicle Communication Protocols for VANETs in the Urban Environment. Future Internet 10(2):14- 14, DOI 10.3390/fi10020014, URL https://dx.doi.org/10.3390/fi10020014.

44. Ahmed E, Gharavi H (2018) Cooperative Vehicular Networking: A Survey. IEEE Transactions on Intelligent Transportation Systems 19(3):996-1014 DOI 10.1109/tits.2018.2795381, URL https://dx.doi.org/10.1109/tits.2018. 2795381.

45. Khan UA, Lee SS (2019) Multi-Layer Problems and Solutions in VANETs: A Review. Electronics 8(2):204-204, DOI 10.3390/electronics8020204, https://dx.doi.org/10.3390/electronics8020204.

46. Storck CR, Duarte-Figueiredo F (2020) A Survey of 5G Technology Evolution, Standards, and Infrastructure Associated With Vehicle-to-Everything Com- munications by Internet of Vehicles. IEEE Access 8:117593-117614, DOI 10.1109/access.2020.3004779, https://dx.doi.org/10.1109/access.2020. 3004779.

47. Gawas MA, Govekar S (2020) State-of-Art and Open Issues of Cross-Layer Design and QOS Routing in Internet of Vehicles. Wireless Personal Com- munications pp 1-37, DOI 10.1007/s11277-020-07790-5, URL https://dx. doi.org/10.1007/s11277-020-07790-5.

48. Ji H, Alfarraj O, Tolba A (2020) Artificial IntelligenceEmpowered Edge of Vehicles: Architecture, Enabling Technologies, and Applications. IEEE Access 8:61020-61034, DOI 10.1109/access.2020.2983609, URL https://dx. doi.org/10.1109/access.2020.2983609.

49. Mitola J, Maguire GQ (1999) Cognitive radio: making software radios more personal. IEEE Personal Communications 6(4):13-18, DOI 10.1109/98. $\quad 788210, \quad$ URL https://dx.doi.org/10.1109/98.788210.
50. Felice MD, Doost-Mohammady R, Chowdhury K, Bononi L (2012) Smart Radios for Smart Vehicles: Cognitive Vehicular Networks. IEEE Vehicular Technology Magazine 7(2):26-33, DOI 10.1109/mvt.2012.2190177.

https://dx.doi.org/10.1109/mvt.2012.2190177.

51. Felice MD, Chowdhury KR, Bononi L (2013), Cognitive radio vehicular ad hoc networks: design, implementation, and future challenges, Mobile Ad Hoc Networking: Cutting Edge Directions, Second Edition 619-644.

52. Singh KD, Rawat P, Bonnin JM (2014) Cognitive radio for vehicular ad hoc networks (CR-VANETs): approaches and challenges. EURASIP Journal on Wireless Communications and Networking 2014(1):1-22, DOI 10.1186/ 1687-1499-2014-49, URL https://dx.doi.org/10.1186/1687-1499-2014-49.

53. Chembe C, Noor RM, Ahmedy I, Oche M, Kunda D, Liu CH (2017) Spectrum sensing in cognitive vehicular network: State-of-Art, challenges and open issues. Computer Communications 97:15-30, DOI 10.1016/j.comcom.2016. 09.002, URL https://dx.doi.org/10.1016/j.comcom.2016.09.002.

54. Silva CM, Masini BM, Ferrari G, Thibault I (2017) A Survey on Infrastructure- Based Vehicular Networks. Mobile Information Systems 2017:1-28, DOI 10.1155/2017/6123868, URL https://dx.doi.org/10.1155/2017/6123868.

55. Kuutti S, Fallah S, Katsaros K, Dianati M, Mccullough F, Mouzakitis A (2018) A Survey of the State-of-the-Art Localization Techniques and Their Potentials for Autonomous Vehicle Applications. IEEE Internet of Things Journal 5(2):829-846, DOI 10.1109/jiot.2018.2812300, URL https://dx.doi. org/10.1109/jiot.2018.2812300.

56. Gerla M, Kleinrock L (2011) Vehicular networks and the future of the mobile internet. Computer Networks 55(2):457-469, DOI 10.1016/j.comnet.2010. 10.015, https://dx.doi.org/10.1016/j.comnet.2010.10.015.

57. Dimitrakopoulos G (2011) Intelligent transportation systems based on internet-connected vehicles: Fundamental research areas and challenges. 11th International Conference on ITS Telecommunications.

58. Imadali S, Kaiser A, Decremps S, Petrescu A, Vèque V (2013) V2V2I: Extended inter-vehicles to infrastructure communication paradigm. Global Information Infrastructure Symposium-GIIS 2013.

59. Tiwari P, Kushwah R (2015) Traffic analysis for VANET using WAVE and WiMAX. International conference on communication networks (ICCN), Gwalior, pp. 343-346.

60. Kaiwartya O, Abdullah AH, Cao Y, Altameem A, Prasad M, Lin CT, Liu X (2016) Internet of vehicles: Motivation, layered architecture, network model, challenges, and future aspects. IEEE Access 4:5356-5373.

61. Apec (2014) White paper of Internet of Vehicles. 50th Telecommunications and information working group meeting, Brisbane, Australia.

62. Hossain E, Niyato D, Han Z (2009) Dynamic spectrum access and management in cognitive radio networks. Cambridge university press.

63. Chao C, Yanmin Z (2013) Augmenting vehicular 3G users through inter- vehicle communications. Wireless Communications and Networking Con- ference (WCNC) pp 1651-1656.

64. Yuan Y, Lei R, Xue L, Xingshe (2013) Delay analysis and study of IEEE $802.11 \mathrm{p}$ based DSRC safety communication in a highway environment. In: Proceedings of the IEEE INFOCOM 2013, pp $1591-1599$

65. Hu G, Huang A, He R, Ai B, Chen Z (2014) Theory analysis of the handover challenge in Express Train Access Networks (ETAN). China Communica- tions 11(7):92-98, DOI 10.1109/cc.2014.6895388, URL https://dx.doi.org/ $10.1109 /$ cc.2014.6895388

66. Datta SK, Harri Z, Bonnet C, Ferreira da Costa R (2017) Vehicles as connected resources: Opportunities and challenges for the future. IEEE Vehicular Technology Magazine 12.2: 26-35.

67. Contreras-Castillo J, Zeadally S, Ibanez JAG (2016) Solving vehicular ad hoc network challenges with big data solutions IET Networks, vol. 5, no. 4, pp. 81-84. 
68. Kamouch A, Chaoub A, Guennoun Z (2018) Mobile big data in vehicular networks: The road to internet of vehicles. Springer, Cham.

69. $\mathrm{Xu} \mathrm{W}$ (2018) Internet of vehicles in big data era. IEEE/CAA Journal of Automatica Sinica 5:19-35.

70. Pourghebleh B, Navimipour NJ (2019) Towards efficient data collection mechanisms in the vehicular ad hoc networks. International Journal of Communication Systems 32(5):e3893e3893, DOI 10.1002/dac.3893, URL https://dx.doi.org/10.1002/dac.3893.

71. Gerla M, Lee EK, Pau G, Lee U (2014) Internet of vehicles: From intelligent grid to autonomous cars and vehicular clouds. IEEE world forum on internet of things.

72. Lee E, Kyu, Gerla M, Pau G, Lee U, Lim JH (2016) Internet of Vehicles: From intelligent grid to autonomous cars and vehicular fogs. International Journal of Distributed Sensor Net- works 12:1550147716665500-1550147716665500.

73. Zhou H, Xu W, Chen J, Wang W (2020) Evolutionary V2X Technologies Toward the Internet of Vehicles: Challenges and Opportunities. Proceedings of the IEEE 108:308-323.

74. Litman T (2017) Autonomous vehicle implementation predictions implications for transport planning,.

75. Litman T (2018) Autonomous vehicle implementation predictions implications for transport planning,.

76. Campbell M, Egerstedt M, How JP, Murray RM (1928) Autonomous driving in urban environments: approaches, lessons and challenges. Philosophical Transactions of the Royal Society of London A: Mathematical, Physical and Engineering Sciences 368:4649-4672

77. 5G-PPP, "5G Automotive Vision," (2015) [Online]. Available: https://5g-ppp.eu/wp-content/uploads/2014/02/5G-PPP-White-

Paper-on-Automotive-Vertical-Sectors.pdf.

78. Department for Transport, "Research on the Impacts of Connected and Autonomous Vehicles (CAVs) on Traffic Flow: Summary Report," (2016) [Online]. Available: https://www.gov.uk/government/uploads/system/uploads/attachm ent_data/file/530091/impacts-of-connected-and-autonomousvehicles-on-traffic-flow-summary-report.pdf.

79. Trösterer S, Meschtscherjakov A, Wilfinger D, Tscheligi M (2014) Eye-tracking in the car: Challenges in a dual-task scenario on a test track. In: Automotive UI '14: 6th International Conference on Automotive User Interfaces and Interactive Vehicular Applications, ACM, pp 1-6.

80. Meschtscherjakov A, Wilfinger D, Murer M, Osswald S, Tscheligi M (2014) Hands-on-the- Wheel: Exploring the design space on the back side of a steering wheel. Ambient Intelligence 8850:299-314.

81. Meschtscherjakov A, Döttlinger C, Rödel M, Tscheligi M (2015) ChaseLight: Ambient LED stripes to control driving speed. In: Proceedings of the 7th International Conference on Automotive User Interfaces and Interactive Vehicular Applications, pp 212219.

82. Grah T, Meschtscherjakov A, Tscheligi M (2016) Dorsal haptic sensory aug- mentation: Fostering drivers awareness of their surroundings with a haptic car seat. Proceedings of the 11th International Conference on Persuasive Technology PERSUASIVE 2016 pp 59-62.

83. Okuda R, Kajiwara Y, Terashima K (2014) A survey of technical trend of adas and autonomous driving. Proceedings of Technical Program - 2014 International Symposium on VLSI Technology, Systems and Application (VLSI-TSA) pp 1-4.

84. Jo K, Kim J, Kim D, Jang C, Sunwoo M (2015) Development of Autonomous Car-Part II: A Case Study on the Implementation of an Autonomous Driving System Based on Distributed Architecture. IEEE Transactions on Industrial Electronics 62(8):5119-5132, DOI 10.1109/tie.2015.2410258, URL https://dx.doi.org/10.1109/tie.2015.2410258.

85. Hussain R, Zeadally.S (2019) Autonomous cars: Research results, issues and future challenges. IEEE Communications Surveys \& Tutorials 21(2):1275-1313, DOI 10.1109/comst.2018.2869360, URL https://dx.doi.org/10.1109/ comst.2018.2869360.

86. Hamid UZ, Abdul H, Zamzuri DK, Limbu (2019) Internet of Vehicle (IoV) Applications in Expediting the Implementation of
Smart Highway of Au- tonomous Vehicle: A Survey. In: Performability in Internet of Things, Springer, pp 137-157.

87. Sadiku MNO, Gupta N, Patel KK, Musa SM (2020) An Overview of Intelligent Transportation Systems in the Context of Internet of Vehicles. Internet of Vehicles and its Applications in Autonomous Driving pp 3-11.

88. Booysen MJ, van Rooyen GJ, Zeadally S (2011) Survey of media access control protocols for vehicular ad hoc networks. IET Communications 5(11):1619- 1631, DOI 10.1049/ietcom.2011.0085, URL https://dx.doi.org/10.1049/ietcom.2011.0085.

89. Booysen T, Gilmore J, Zeadally S, Rooyen J (2012) Machine-toMachine (M2M) communications in vehicular networks. KSII Transactions on In- ternet and Information Systems 6(2):529-546.

90. Omar HA, Zhuang W, Li L (2013) VeMAC: A TDMA-Based MAC Protocol for Reliable Broadcast in VANETs. IEEE Transactions on Mobile Computing 12(9):1724-1736, DOI 10.1109/tmc.2012.142, URL https://dx.doi.org/10.1109/tmc.2012.142.

91. Bharati S, Zhuang W (2013) CAH-MAC: Cooperative ADHOC MAC for Vehicular Networks. IEEE Journal on Selected Areas in Communications 31(9):470-479, DOI 10.1109/jsac.2013.sup.0513042, URL https://dx.doi. org/10.1109/jsac.2013.sup.0513042.

92. Huang JM (2013) Research on Internet of Vehicles and its Application in Intelligent Transportation. Applied Mechanics and Materials 321-324:2818- 2821, DOI 10.4028/www.scientific.net/amm.321-324.2818, URL https://dx. doi.org/10.4028/www.scientific.net/amm.321-324.2818.

93. Lu N, Cheng N, Zhang N, Shen X, Mark JW (2014) Connected vehicles: Solutions and challenges. IEEE internet of things journal 1:289-299.

94. Wu W, Yang Z, Li K (2016) Internet of vehicles and applications, Internet of Things. Morgan Kaufmann, 299-317.

95. Qu F, Wu Z, Wang FY, Cho W (2015) A security and privacy review of vanets. IEEE Transactions on Intelligent Transportation Systems 16:2985-2996.

96. Azees M, Deborah LJ, Vijayakumar P (2016) Comprehensive survey on security services in vehicular ad-hoc networks. IET Intelligent Transport Systems, 10(6):379-388, DOI 10.1049/ietits.2015.0072, URL https://dx.doi. org/10.1049/iet-its.2015.0072.

97. Ning Z, Kwok RYK, Zhang K, Wang X, Obaidat MS, Guo L, Hu X, Hu B, Guo Y, Sadoun B (2020) Joint Computing and Caching in 5G-Envisioned Internet of Vehicles: A Deep Reinforcement Learning-Based Traffic Control System. IEEE Transactions on Intelligent Transportation Systems pp 1-12, DOI 10.1109/tits.2020.2970276, https://dx.doi.org/10.1109/tits.2020. 2970276.

98. Pavan B, Sri M, Mahesh VP, Harigovindan (2020) IEEE 802.11 ah for Internet of Vehicles: Design Issues and Challenges. Internet of Vehicles and its Applications in Autonomous Driving, Springer, Cham 41-61.

99. Woo S, Jo HJ, Lee DH (2014) A practical wireless attack on the connected car and security protocol for in-vehicle can. IEEE Transactions on Intelligent Transportation Systems 16(2):9931006.

100. Woo S, Jo HJ, Kim IS, Lee DH (2016) A practical security architecture for in-vehicle can-fd. IEEE Transactions on Intelligent Transportation Systems 17:2248-2261.

101. Bhardwaj I, Khara S (2018) Research trends in Architecture, Security, Services and Applications of Internet of Vehicles (IOV). 2018 International Conference on Computing.

102. Groza B, Murvay PS (2018) Security Solutions for the Controller Area Net- work: Bringing Authentication to In-Vehicle Networks. IEEE Vehicular Technology Magazine 13(1):40-47, DOI 10.1109/mvt.2017.2736344, https://dx.doi.org/10.1109/mvt.2017.2736344.

103. Asuquo P (2018) Security and Privacy in Location-Based Services for Vehicular and Mobile Communications: An Overview, Challenges, and Countermea- sures. IEEE Internet of Things Journal 5:4778-4802. 
104. Boumerdassi S, Ã Renault (2016) A flooding-based solution to improve loca- tion services in vanets. 2016 IEEE International Conference on Communi- cations (ICC) pp 1-6.

105. Aissaou R, Dhraief A, Belghith A, Menouar H, Filali F, Mathkour $H$ (2016) Vals: Vehicle-aided location service in urban environment. 2016 IEEE Wire- less Communications and Networking Conference pp 1-6.

106. Banani S, Goron S (2014) Selecting basic safety messages to verify in VANETs using zone priority. The 20th Asia-Pacific Conference on Pattaya pp 423-428.

107. Hamida EB, Javed MA (2016) Channel-aware ECDSA signature verification of basic safety mess- ages with k-means clustering in VANETs. Proceedings of IEEE International Conference on Advanced Information Networking and Applications pp 1-8.

108. Sun Y (2015) Security and Privacy in the Internet of Vehicles. 2015 Inter- national Conference on Identification, Information, and Knowledge in the Internet of Things (IIKI).

109. Bariah L, Shehada D, Salahat E, Yeun CY (2015) Recent advances in vanet security: A survey. 2015 IEEE 82nd Vehicular Technology Conference (VTC2015-Fall) pp 1-7.

110. Papadimitratos $P$ (2016) Security on wheels: Security and privacy for vehicular communication systems. In: Proceedings of the 2016 ACM SIGSAC Conference on Computer and Communications Security, CCS '16, ACM, pp 1855-1856.

111. Sharma S, Kaushik B (2020) A Comprehensive Review of Natureinspired Algorithms for Internet of Vehicles. 2020 International Conference on Emerging Smart Computing and Informatics (ESCI).

112. Contreras-Castillo J, Zeadally S, Ibáñez JAG (2017) A sevenlayered model architecture for Internet of Vehicles. Journal of Information and Telecommunication 1(1):4-22, DOI 10.1080/24751839.2017.1295601, URL https://dx.doi.org/10.1080/24751839.2017.1295601.

113. Contreras-Castillo J, Zeadally S, Guerrero-Ibanez JA (2018) Internet of Vehicles: Architecture, Protocols, and Security. IEEE Internet of Things Journal 5(5):3701-3709, DOI 10.1109/jiot.2017.2690902, https://dx.doi.org/10.1109/jiot.2017.2690902.

114. Qureshi KN, Din S, Jeon G, Piccialli F (2020) Internet of Vehicles: Key Technologies, Network Model, Solutions and Challenges With Future Aspects. IEEE Transactions on Intelligent Transportation Systems pp 1-10, DOI 10. 1109/tits.2020.2994972, URL https://dx.doi.org/10.1109/tits.2020.2994972.

115. Tuyisenge L (2018) Network Architectures in Internet of Vehicles (IoV): Re- view, Protocols Analysis, Challenges and Issues. In: International Confer- ence on Internet of Vehicles, Springer.

116. Ang LM, Seng KP, Ijemaru GK, Zungeru AM (2019) Deployment of IoV for Smart Cities: Applications, Architecture, and Challenges. IEEE Access 7:6473-6492, DOI 10.1109/access.2018.2887076, https://dx.doi.org/10.1109/access.2018.2887076.

117. Hao W, Palekar M, Fujimoto R, Guenslar R, Hunter M, Lee J, Ko J (2005) An empirical study of short range communications for vehicles, 83-84.

118. Hao W, Lee J, Hunter M, Fujimoto R (2005) Efficiency of simulated vehicle-to-vehicle message propagation in Atlanta, Georgia, I-75 corridor, Transportation research record 1910.1:8289.

119. Dutta AK, Elhoseny M, Dahiya V, Shankar K (2020) An efficient hierarchi- cal clustering protocol for multihop Internet of vehicles communication. Transactions on Emerging Telecommunications Technologies 31(5):3690 - 3690, DOI 10.1002/ett.3690, URL https://dx.doi.org/10.1002/ett.3690.

120. Korkmaz G, Ekici E, Ozguner F (2006) Internet access protocol providing QoS in vehicular networks with infrastructure support. IEEE.

121. Du L, Ukkusuri S, Del Valle WF, Kalyanaraman S (2009) Optimization models to characterize the broadcast capacity of vehicular ad hoc networks. Transportation Research Part C: Emerging Technologies 17:571-585.
122. Lv P, Wang X, Xue X, Xu M (2015) SWIMMING: Seamless and efficient WiFi-based Internet access from moving vehicles. IEEE Transactions on Mobile Computing 14:1085- 1097.

123. Tuyisenge L, Ayaida M, Tohme S, Afilal LE (2020) Handover Mechanisms in Internet of Vehicles (IoV): Survey, Trends, Challenges, and Issues. Global Advancements in Connected and Intelligent Mobility: Emerging Research and Opportunities pp 1-64.

124. Zhang S, Liu E (2015) Ubiquitous Computing and Communications; Depend- able, Autonomic and Secure Computing; Pervasive Intelligence and Com- puting. 2015 IEEE International Conference on Computer and Information Technology.

125. Eze E, Zhang S, Liu E, Eze J, Muhammad S (2018) Reliable and enhanced cooperative cross-layer medium access control scheme for vehicular commu- nication. IET Networks 7(4):200-209, DOI 10.1049/iet-net.2017.0232, URL https://dx.doi.org/10.1049/ietnet.2017.0232.

126. Eze EC, Zhang S, Liu E, Nweso EN, Eze JC (2016) Timely and reliable packets delivery over internet of vehicles for road accidents prevention: a cross-layer approach. IET Networks 5(5):127-135, DOI 10.1049/iet-net.2015.0112, URL https://dx.doi.org/10.1049/iet-net.2015.0112.

127. Namritha R, Karuppanan K (2011) Opportunistic dissemination of emergency messages using VANET on urban roads. 2011 International Conference on Recent Trends in Information Technology (ICRTIT).

128. Jin M, Zhou X, Luo E, Qing X (2015) Industrial-QoS-oriented remote wireless communication protocol for the Internet of construction vehicles. IEEE Transactions on Industrial Electronics 62:7103-7113.

129. Tropea M, Santamaria AF (2015) Vehicular Traffic Optimization in VANETs: a Proposal for Nodes Re-routing and Congestion Reduction. Advances in Electrical and Electronic Engineering 13(4):376-385, DOI 10.15598/aeee. v13i4.1495, URL https://dx.doi.org/10.15598/aeee.v13i4.1495.

130. Mersky AC, Samaras C (2016) Fuel economy testing of autonomous vehicles. Transportation Research Part C: Emerging Technologies 65:31-48, DOI 10. 1016/j.trc.2016.01.001, URL https://dx.doi.org/10.1016/j.trc.2016.01.001.

131. He X, Ren Z, Shi C, Fang J (2016) A novel load balancing strategy of software-defined cloud/fog networking in the Internet of Vehicles. China Communications 13(Supplement2):140-149, DOI 10.1109/cc.2016.7833468, https://dx.doi.org/10.1109/cc.2016.7833468.

132. Fabian P, Rachedi A, Guéguen C (2020) Programmable objective function for data transportation in the Internet of Vehicles. Transactions on Emerging Telecommunications Technologies 31(5):3882-3882, DOI 10.1002/ett.3882,URL https://dx.doi.org/10.1002/ett.3882.

133. Fabian P (2018) Fuzzy-based objective function for routing protocol in the internet of things. IEEE Global Communications Conference (GLOBECOM).

134. Huang W, Ding L, Meng D, Hwang JN, Xu Y, Zhang W (2018) QoE-based resource allocation for heterogeneous multi-radio communication in software-defined vehicle networks. IEEE Access 6:3387-3399.

135. Yuan Q, Li J, Liu Z, Yang F (2016) Space and time constrained data offloading in vehicular net- works. IEEE 18th International Conference on High Performance Comput- ing and Communications; IEEE 14th International Conference on Smart City; IEEE 2nd International Conference on Data Science and Systems (HPCC/SmartCity/DSS).

136. Davydov V, Bezzateev S (2020) Accident Detection in Internet of Vehicles using Blockchain Technology. 2020 International Conference on Information Networking (ICOIN).

137. Chang B, Chen J, Hsieh C (2009) Markov decision process-based adaptive vertical handoff with RSS prediction in heterogeneous wireless networks. Wireless communications and networking conference (WCNC) pp 1-6.

138. Kunarak S, Seleesathira R (2010) Predictive RSS with fuzzy logic based vertical handoff algorithm in heterogeneous wireless networks. In: The 2010 International symposium on 
communications and information technologies (ISCIT), pp 189194.

139. Guerrero-Ibáñez A, Contreras-Castillo J, Barba A, Reyes A (2011) A QoS- based dynamic pricing approach for services provisioning in heterogeneous wireless access networks. Pervasive and Mobile Computing 7(5):569-583, DOI 10.1016/j.pmcj.2010.10.003, URL https://dx.doi.org/10.1016/j.pmcj. 2010.10.003.

140. Guerrero-Ibanez JA, Zeadally S, Contreras-Castillo J (2015) Integration challenges of intelligent transportation systems with connected vehicle, cloud computing, and internet of things technologies. DOI 10.1109/mwc.2015. 7368833, URL https://dx.doi.org/10.1109/mwc.2015.7368833.

141. Bitam S, Mellouk A, Zeadally S (2015) VANET-cloud: a generic cloud computing model for vehicular Ad Hoc networks. IEEE Wireless Communications 22(1):96-102, DOI 10.1109/mwc.2015.7054724, https://dx.doi.org/10.1109/mwc.2015.7054724.

142. de la Iglesia I, Hernandez-Jayo U, Osaba E, Carballedo R (2017) Smart Bandwidth Assignation in an Underlay Cellular Network for Internet of Vehicles. Sensors 17(10):2217-2217, DOI 10.3390/s17102217, URL https://dx.doi.org/10.3390/s17102217.

143. Chen C, Liu X, Qiu T, Liu L, Sangaiah AK (2017) Latency estimation based on traffic density for video streaming in the internet of vehicles. Computer Communications 111:176-186.

144. Kumar PM, G UD, Manogaran G, Sundarasekar R, Chilamkurti N, Varatharajan R (2018) Ant colony optimization algorithm with Internet of Vehicles for intelligent traffic control system. Computer Networks 144:154-162, DOI 10.1016/j.comnet.2018.07.001, URL https://dx.doi.org/10.1016/ j.comnet.2018.07.001.

145. Alzamzami O, Mahgoub I (2019) Fuzzy Logic-Based Geographic Routing for Urban Vehicular Networks Using Link Quality and Achievable Through- put Estimations. IEEE Transactions on Intelligent Transportation Systems 20(6):2289-2300, DOI 10.1109/tits.2018.2867177, https://dx.doi.org/10.1109/tits.2018.2867177.

146. Wu Z, Lu Z, Hung PC, Huang SC, Tong Y, Wang Z (2019) QaMeC: A QoS-driven IoVs application optimizing deployment scheme in multimedia edge clouds. Future Generation Computer Systems 92:17-28.

147. Venkatramana DKN, Srikantaiah SB, Moodabidri J (2018) CISRP: connectivity-aware intersection-based shortest path routing protocol for VANETs in urban environments. IET Networks 7(3):152-161, DOI 10.1049/ iet-net.2017.0012, URL https://dx.doi.org/10.1049/iet-net.2017.0012.

148. Zhang G, Wu M, Duan W, Huang X (2018) Genetic Algorithm Based QoS Perception Routing Protocol for VANETs. Wireless Communications and Mobile Computing'

149. Su, Zhou, Hui Y, Xu Q, Yang T, Liu J, Jia Y (2018) An edge caching scheme to distribute content in vehicular networks. IEEE Transactions on Vehicular Technology 67:5346-5356.

150. Mouhcine E, Mansouri K, Mohamed Y (2018) Solving Traffic Routing System using VANet Strategy Combined with a Distributed Swarm Intelligence Optimization.

151. Philip BV, Alpcan T, Jin J, Palaniswami M (2019) Distributed Real-Time IoT for Autonomous Vehicles. IEEE Transactions on Industrial Informatics 15(2):1131-1140, DOI 10.1109/tii.2018.2877217, https://dx.doi.org/10.1109/tii.2018.2877217.

152. Sherazi HHR, Khan ZA, Iqbal R, Rizwan S, Imran MA, Awan K (2019) A Heterogeneous IoV Architecture for Data Forwarding in Vehicle to Infrastructure Communication. Mobile Information Systems 2019:1-12, DOI 10.1155/2019/3101276, URL https://dx.doi.org/10.1155/2019/3101276.

153. Wang CCR, Lien JJJ (2008) Automatic Vehicle Detection Using Local Features-A Statistical Approach. IEEE Transactions on Intelligent Trans- portation Systems 9(1):83-96, DOI 10.1109/tits.2007.908572, https://dx.doi.org/10.1109/tits.2007.908572

154. Uppoor S, Fiore M (2015) Characterizing Pervasive Vehicular Access to the Cellular RAN Infrastructure: An Urban Case Study. IEEE Transactions on Vehicular Technology 64(6):2603-2614,
DOI $\quad 10.1109 /$ tvt.2014.2343651,

URL

https://dx.doi.org/10.1109/tvt.2014.2343651.

155. Martínez-Vidal R, Martí R, Sreenan CJ, Borrell J (2016) Measuring QoS in an Aeronautical Opportunistic Network Architecture with Limited Ac- cess to a Satellite Communications Backhaul. Mobile Information Systems 2016:1-12, DOI 10.1155/2016/7601316, URL https://dx.doi.org/10.1155/2016/7601316.

156. Bauza R, Gozalvez J (2013) Traffic congestion detection in large-scale scenarios using vehicle-to-vehicle communications. Journal of Network and Computer Applications 36(5):1295-1307, DOI 10.1016/j.jnca.2012.02.007, https://dx.doi.org/10.1016/j.jnca.2012.02.007.

157. Park S, Kim J, Lee S, Hwang K (2017) Experimental analysis on control constraints for connected vehicles using vissim. International Symposia of Transport Simulation (ISTS) and the International Workshop on Traffic Data Collection and its Standardization (IWTDCS) 21:269-280.

158. Lu N, Zhang N, Cheng N, Shen X, Mark JW, Bai F (2013) Vehicles meet infrastructure: Toward capacity-cost tradeoffs for vehicular access networks. IEEE transactions on intelligent transportation systems 14:1266-1277.

159. Salahuddin MA, Al-Fuqaha A, Guizani M (2015) Software-Defined Networking for RSU Clouds in Support of the Internet of Vehicles. IEEE Internet of Things Journal 2(2):133-144, DOI 10.1109/jiot.2014.2368356, https://dx.doi.org/10.1109/jiot.2014.2368356.

160. Ligo AK, Peha JM, Ferreira P, Barros J (2018) Throughput and Economics of DSRC-Based Internet of Vehicles. IEEE Access 6:7276-7290, DOI 10.1109/ access.2017.2785499, URL https://dx.doi.org/10.1109/access.2017.2785499.

161. Wang W, Xia F, Nie H, Chen Z, Gong Z, Kong X, Wei W (2020) Vehicle trajectory clustering based on dynamic representation learning of Internet of vehicles. IEEE Transactions on Intelligent Trans- portation Systems

162. Kamgueu PO, Nataf E, Djotio TN (2015) On design and deployment of fuzzy-based metric for routing in low-power and lossy networks. IEEE 40th Local Computer Networks Conference Workshops (LCN Workshops.

163. Rios M, Gondim VL, Monteiro PC, C (2012) Use of fuzzy logic for networks selection in heterogeneous wireless environment. In: 14th International Conference on Advanced Communication Technology (ICACT), pp 798-803.

164. Zhang D, Zhang Y, Lv N, He Y (2013) An access selection algorithm based on GRA integrated with FAHP and entropy weight in hybrid wireless environment. In: 7th International Conference on application of information and communication technologies (AICT), pp 1-5.

165. Nguyen AT, Sentouh C, Popieul JC (2018) Fuzzy steering control for autonomous vehicles under actuator saturation: Design and experiments. Journal of the Franklin Institute 355(18):9374-9395, DOI 10.1016/j.jfranklin. 2017.11.027, URL https://dx.doi.org/10.1016/j.jfranklin.2017.11.027.

166. Lamaazi H, Benamar N (2018) OF-EC: A novel energy consumption aware objective function for RPL based on fuzzy logic. Journal of Network and Computer Applications 117:42-58, DOI 10.1016/j.jnca.2018.05.015, URL https://dx.doi.org/10.1016/j.jnca.2018.05.015.

167. Lamaazi H, Ahmadi AE, Benamar N, Jara AJ (2019) OF-ECF: A New Optimization of the Objective Function for Parent Selection in RPL. 2019 International Conference on Wireless and Mobile Computing, Networking and Communications (WiMob) pp 27-32.

168. Priyan MK, Devi GU (2018) Energy efficient node selection algorithm based on node performance index and random waypoint mobility model in internet of vehicles. Cluster Computing 21(1):213-227, DOI 10.1007/s10586-017-0998- x, URL https://dx.doi.org/10.1007/s10586-017-0998-x.

169. Mukherjee, Priyadarshi, and Swades De. "cDIP: channel-aware dynamic window protocol for energy-efficient IoT communications." IEEE Internet of Things Journal 5.6 (2018): 4474-4485. 
170. Zhang T, Zheng L, Jiang Y, Tian Z, Du X, Guizani M (2020) A method of chained recommendation for charging piles in internet of vehicles. Computing pp 1-19.

171. Chiti F, Fantacci R, Gu Y, Han Z (2017) Content sharing in Internet of Vehicles: Two matching-based user-association approaches. Vehicular Communications 8:35-44, DOI 10.1016/j.vehcom.2016.11.005, URL https://dx.doi.org/10.1016/j.vehcom.2016.11.005.

172. Chaqfeh M, Mohamed N, Jawhar I, Wu J (2016) Vehicular cloud data collection for intelligent transportation systems, 2016 3rd Smart Cloud Networks and Systems (SCNS).

173. Amadeo M, Campolo C, Quevedo J, Corujo D, Molinaro A, Iera A, Aguiar RL, Vasilakos AV (2016) Information-centric networking for the internet of things: challenges and opportunities. IEEE Network 30:92-100.

174. Chen H, Zhang X, Liu GP (2007) Simulation and visualization of empirical traffic models using vissim. 2007 IEEE International Conference on Net- working, Sensing and Control pp 879-882.

175. Papageorgiou M, Papamichail I, Messmer A, Wang Y (2010) Traffic Simulation with METANET. Springer, New York, NY; New York.

176. Sommer C, German R, Dressler F (2011) Bidirectionally Coupled Network and Road Traffic Simulation for Improved IVC Analysis. IEEE Transactions on Mobile Computing 10(1):3-15.

177. Cho W, Kim SH (2017) Multimedia Sensor Dataset for the Analysis of Vehicle Movement. Proceedings of the 8th ACM on Multimedia Systems Conference.

178. Xie Y, Su X, He Y, Chen X, Cai G, Xu B, Ye W (2017) Stm32based vehicle data acquisition system for internet-of- vehicles. 2017 IEEE/ACIS 16th International Conference on Computer and Information Science (ICIS).

179. Liu Y, Cheng D, Wang Y, Cheng J, Gao S (2018) A Novel Method for Predicting Vehicle State in Internet of Vehicles. Mobile Information Systems2018.

180. Yu F, Xian W, Chen Y, Liu F, Liao M, Madhavan V, Darrell T (2018) BDD100K: A diverse driving video database with scalable annotation tooling." arXiv preprint arXiv:1805.04687.

181. Smitha A, Pai MM, Ajam N, Mouzna J (2013) An optimized adaptive algorithm for authentication of safety critical messages in VANET. 8th International Conference on Communications and Networking in China (CHINACOM).

182. Yang S, Liu Z, Li J, Wang S, Yang F (2016) Anomaly detection for Internet of Vehicles: A trust management scheme with affinity propagation. Mobile Information Systems.

183. Makarfi AU, Rabie KM, Kaiwartya O, Adhikari K, Nauryzbayev G, Li X, Kharel R (2020) Towards Physical Layer Security for Internet of Vehicles: Interference Aware Modelling. IEEE Internet of Things Journal pp 1-1, DOI 10.1109/jiot.2020.3006527, URL https://dx.doi.org/10.1109/jiot.2020. 3006527.

184. Kang J, Yu R, Huang X, Jonsson M, Bogucka H, Gjessing S, Zhang $Y$ (2016) Location privacy attacks and defenses in cloud-enabled internet of vehicles. IEEE Wireless Communications 23:52-59.

185. Joy J, Gerla M (2017) Internet of vehicles and autonomous connected car- privacy and security issues. 26th International Conference on Computer Communication and Networks (ICCCN).

186. Pokhrel SR, Choi J (2020) Improving TCP Performance Over WiFi for Internet of Vehicles: A Federated Learning Approach. IEEE Transactions on Vehicular Technology 69(6):6798-6802, DOI 10.1109/tvt.2020.2984369, https://dx.doi.org/10.1109/tvt.2020.2984369.

187. Xu C, Liu H, Zhang Y, Wang P (2020) Mutual authentication for vehicular network in complex and uncertain driving. Neural Computing and Applications pp 1-12.

188. Ahmed S, Kumari S, Saleem MA, Agarwal K, Mahmood K, Yang MH (2020) Anonymous Key-Agreement Protocol for V2G Environment Within Social Internet of Vehicles. IEEE Access 8:119829-119839.

189. Zhou H, Xu W, Chen J, Wang W (2020) Evolutionary V2X technologies toward the Internet of vehicles: Challenges and opportunities. Proceedings of the IEEE 108:308-323.

190. "Corsim: Microscopic traffic simulation model." URL http://www-mctrans.ce.ufl.edu/featured/tsis/version5/ corsim.htm.
191. "Transportation analysis and simulation system (transims)." http:// ndssl.vbi.vt.edu/apps/transims.htm.

192. "Paramics: Microscopic traffic simulation." URL https://www.epcc.ed.ac.uk/projects-portfolio/ paramicsmicroscopic-traffic-simulation.

193. https://www.tataelxsi.com/V2X-Emulator/index.html 